\title{
Zero modes in magnetic systems: General theory and an efficient computational scheme
}

\author{
F. J. Buijnsters, * A. Fasolino, and M. I. Katsnelson \\ Institute for Molecules and Materials, Radboud University Nijmegen, Heyendaalseweg 135, 6525 AJ Nijmegen, Netherlands
}

(Received 4 December 2013; published 30 May 2014)

\begin{abstract}
The presence of topological defects in magnetic media often leads to normal modes with zero frequency (zero modes). Such modes are crucial for long-time behavior, describing, for example, the motion of a domain wall as a whole. Conventional numerical methods to calculate the spin-wave spectrum in magnetic media are either inefficient or they fail for systems with zero modes. We present a new efficient computational scheme that reduces the magnetic normal-mode problem to a generalized Hermitian eigenvalue problem also in the presence of zero modes. We apply our scheme to several examples, including two-dimensional domain walls and Skyrmions, and show how the effective masses that determine the dynamics can be calculated directly. These systems highlight the fundamental distinction between the two types of zero modes that can occur in spin systems, which we call special and inertial zero modes. Whereas the inertial modes are generic Goldstone modes related to a broken continuous symmetry, the special modes arise naturally when two broken continuous symmetries coexist. Our method is suitable for both conservative and dissipative systems. For the latter case, we present a perturbative scheme to take into account damping, which can also be used to calculate dynamical susceptibilities.
\end{abstract}

DOI: 10.1103/PhysRevB.89.174433

\section{INTRODUCTION}

Many properties of magnetic systems can be understood at the classical level by studying their magnetic structure and behavior on the submicron length scale (micromagnetics [1-3]) or atomistically (atomistic spin dynamics [4,5]). In these approaches, the dynamics of the microscopic magnetic moments is described by the Landau-Lifshitz-Gilbert (LLG) equation [6,7]. The various competing interactions (exchange, anisotropy, dipolar, Zeeman,...) in micromagnetic models often result in a rich energy landscape with multiple local energy minima and hysteresis [1,8]. Nontrivial magnetic configurations may be very stable, for instance, if they contain topological defects such as domain walls or magnetic Skyrmion bubbles [8,9].

It is often useful to study the dynamics of small-amplitude deviations from a given magnetic equilibrium configuration (linearization). The eigenmodes of the linearized LLG equation are known as magnetic normal modes. In homogeneous systems, the magnetic normal modes are spin waves, which propagate through the material $[10,11]$. The presence of inhomogeneities, whether intrinsic (lattice defects, boundaries) or configurational (domain walls, Skyrmions), changes this picture. Such defects do not only affect the dynamics of the spin waves; they also often give rise to special low-energy normal modes that are localized near the defect [12-14]. The modes localized on configurational defects are particularly interesting. They provide valuable insight into the dynamics of domain walls [15] and other topological defects [16], a sound understanding of which will be important for the development of novel magnetic-storage technologies such as racetrack memory [17]. The low-energy modes also provide a channel for dissipation [18,19]. Microscopic magnetic elements, such as ferromagnetic rings, are another class of systems with potential for technological application [20]. The spin-wave mode spectrum of these elements can be determined

*f.buijnsters@ science.ru.nl
PACS number(s): 75.78.Cd, 75.78.Fg, 45.20.Jj, 45.30.+s experimentally using magnetic-response measurements or Brillouin light scattering, providing a very direct test of micromagnetic models [20-23].

Zero-frequency modes (zero modes) arise naturally in the presence of broken continuous symmetries. We shall see that for spin systems, such modes require special consideration both from a theoretical and a numerical point of view. This work introduces a general classification, based on the theory of linear Hamiltonian systems [24], of the normal modes in spin systems, which we shall call positive modes, special zero modes, and inertial zero modes. We shall see that the inertial zero modes correspond to a broken continuous symmetry, while special zero modes may arise when two broken continuous symmetries coexist. Both types of zero modes can be associated with a (quasi)continuous spectrum of low-energy excitations in extended systems (Goldstone's theorem). It turns out that the qualitative shape of the dispersion relation of the Goldstone mode depends on whether the zero mode is special or inertial.

While exact or approximate analytical solutions of the magnetic normal-mode problem do exist in certain special cases $[12,13,20]$, in general it can be solved only numerically. In some cases, the magnetic normal modes can be obtained by a "brute-force" method: numerically integrating the LLG equation over a certain time interval and performing a fast Fourier transformation (FFT) in the time domain [14,20,25,26]. While in principle effective, this approach is limited to relatively small systems by the large amounts of CPU power and memory storage it requires, especially if a good frequency resolution is to be achieved (long simulation times). Moreover, it requires some manual tuning (reasonable settings for the initial amplitudes and sampling frequencies) and it fails to detect zero-frequency and degenerate modes. In addition to the theoretical analysis, this work presents a direct numerical procedure that can be used to find the magnetic normal modes of any spin system near any given equilibrium configuration (more precisely, near any local energy minimum). It can deal efficiently and scalably with any type of interaction, including long-range interactions, and does not assume that the 
material is homogeneous or that the equilibrium configuration is collinear [27].

An efficient numerical approach should somehow be based on a direct calculation of the eigenvectors and eigenvalues of the dynamical matrix that results from linearization of the LLG equation [28]. However, we shall see that this dynamical matrix is not necessarily diagonalizable, so that eigenvectors in the usual sense may not even exist. Diagonalizability can only be guaranteed if no zero modes are present. To the best of our knowledge, this fact has been overlooked in all previous works describing general methods for the magnetic normal-mode problem [27-31]. While there certainly are many cases in which this issue does not occur [21,22,28,30,32-38] or occurs for a very special choice of parameters [39-41], we shall see that zero modes appear in many relevant physical systems. Of course, finite-size effects and magnetostatic interactions mean that the Hamiltonian is usually only approximately invariant under the relevant continuous symmetry. However, we argue that even in those cases it is useful first to calculate the modes of the topological defect in a system with perfect translational (or other) symmetry in order to investigate the equations of motion and the dynamical parameters. In such situations, zero modes are an essential part of the analysis. Indeed, precisely the zero modes are often the most important for the dynamics of topological defects. For example, we shall see that it is the zero modes that determine whether the dynamics of a topological defect is inertial and, if so, with what effective mass.

Our method has a firm basis in the general theory of Hamiltonian systems [24]. We show that the normal-mode problem of an arbitrary (conservative) Hamiltonian system at a local energy minimum can be cast in the form of a Hermitian definite generalized eigenvalue problem (HDGEP) [42], Dx = $\lambda S x$, where the matrices $D$ and $S$ are Hermitian and $S$ is positive definite, which can be solved particularly efficiently. The most popular methods for large eigenvalue problems (Lanczos, conjugate-gradient nonlinear optimization, ....) require the problem to be of this form. Important features of these methods are that they operate in an incremental fashion (the lowest modes are calculated first) and that they can be implemented in a matrix-free manner [43] (they are Krylov-subspace methods [44]). These features make the HDGEP methods considerably scalable. First, the low modes of a very large system, which are often the most physically relevant, may be obtained without solving the full eigenvalue problem for all eigenvectors, which would obviously take at least $\mathcal{O}\left(N^{2}\right)$ time. Second, it is not necessary to store the interaction matrix in explicit form, which will contain $\mathcal{O}\left(N^{2}\right)$ nonzero values if the long-range dipolar interactions are taken into account. It is sufficient to provide a routine that evaluates the forces or torques for any given specific configuration. When implemented using FFT or multigrid techniques, such a routine can run in $\mathcal{O}(N \log N)$ instead of $\mathcal{O}\left(N^{2}\right)$ time [29].

We obtain a solution method for the normal-mode problem of the conservative (zero damping) spin system as an immediate special case of our method for general Hamiltonian systems. A similar reduction of the conservative magnetic normal-mode problem to the HDGEP was proposed in Refs. [31] and [45] by assuming that the Hessian matrix of the Hamiltonian is positive definite at a local minimum, which is not always the case. Reference [45] raised this issue explicitly but did not provide a solution. We now solve this problem and introduce a systematic classification and a numerical method that also work if the Hessian matrix of the Hamiltonian at the equilibrium configuration is not positive definite but merely positive semidefinite (also called non-negative definite), as it is in the presence of zero modes. An additional advantage of our method is that it may be used directly in Cartesian coordinates, in which the micromagnetic Hamiltonians normally take a very simple form (often quadratic). We do not need to go over to spherical coordinates, which are more computationally expensive and have singularities at certain points.

For the spin system with damping, we derive explicit expressions for the normal modes by treating the damping term of the LLG equation as a perturbation. In this way we can obtain the damped modes and decay rates to a good approximation without the need for solving non-Hermitian eigenvalue problems.

This paper is organized as follows. In Sec. II, we state some general properties of the normal modes of linearized Hamiltonian systems that are essential for what follows. Here we introduce the nomenclature of special and inertial zero modes and specify their distinct dynamics. A more detailed discussion is provided in the Appendix. In Sec. III, we make the definitions of Sec. II explicit for the conservative spin system. Section IV then shows how the normal-mode problem of a Hamiltonian system, such as the conservative spin system, near a local energy minimum can be reduced to the HDGEP. We specifically show how to deal with zero modes in a robust way. We present perturbative expressions for the spin system with damping in Sec. V. These expressions can be used to calculate dynamical susceptibility functions (Sec. VI). Section VII explains how the method can be efficiently implemented in a computer code. Section VIII provides examples of magnetic normal modes in various spin systems, highlighting some key features of magnetic normal modes. Normal modes provide a very convenient starting point for a collective-coordinate analysis of the dynamics of topological defects. In Sec. VIII B, we focus on the two qualitatively different types of effective dynamical behavior (inertial and noninertial) that may be found when a magnetic equilibrium configuration containing some (topological) defect is perturbed by an external force. We show how a normal-mode analysis that includes zero modes immediately provides the equations of motion and effective masses of such magnetic structures. In Sec. VIII D we discuss in detail the relation between the Goldstone zero modes (special and inertial) and the corresponding branches of low-energy excitations. We apply our framework to the excitations of an extended domain wall and draw an analogy to phonons in two-dimensional crystals such as graphene. Section IX summarizes our results.

\section{NORMAL MODES OF HAMILTONIAN SYSTEMS}

This section states some results from the theory of Hamiltonian systems that are essential for the following sections. In particular, we introduce our nomenclature for the three types of normal modes (positive, special zero, inertial zero) that may appear in systems with a positive semidefinite Hamiltonian. A more thorough discussion with explanations and references is provided in the Appendix. 
Let us consider a time-invariant dynamical system near an equilibrium point, which we take to lie at $x=0$. Its equation of motion is given by

$$
\dot{x}^{i}=M^{i}{ }_{j} x^{j}+\mathcal{O}\left(\|x\|^{2}\right),
$$

where $x^{1}, \ldots, x^{m}$ represent a nonsingular system of coordinates and the dot denotes the time derivative. Our goal is to find the eigenvalues and eigenvectors of $M$. This cannot normally be accomplished by a diagonalization of $M$, because (a) in general, $M$ is very large but not symmetric, so that the efficient iterative methods for the HDGEP cannot be used; and (b) $M$ might not be diagonalizable at all (it may be defective). However, if the dynamical system (1) is a linear or nonlinear Hamiltonian system, we shall see that we can bypass these problems by introducing a certain antisymmetric matrix $\Omega$. The elements of $\Omega$ are given by

$$
\Omega^{i j}=-\left.\left\{x^{i}, x^{j}\right\}\right|_{x=0}=\left.\left\{x^{j}, x^{i}\right\}\right|_{x=0},
$$

the value at the equilibrium point of the Poisson bracket between the coordinates $x^{j}$ and $x^{i}$. It can be shown (see the Appendix) that for a Hamiltonian system, the matrix $M$ is such that $M \Omega$ is symmetric.

For certain physical systems, Hamiltonian dynamics takes place only on a subspace of the space where the coordinates are defined. An example is the spin system: While a magnetic moment $\mathbf{m}$ is defined on $\mathbb{R}^{3}$, its dynamics is restricted to a subset of the form $\left\{\mathbf{m} \in \mathbb{R}^{3}:\|\mathbf{m}\|=c\right\}$ for some $c \geqslant 0$. The dimension of this "accessible subspace" (symplectic leaf [46]) is always even. For a system of $n$ spins in Cartesian coordinates, we have $m=3 n$, while the dimension of the symplectic leaf is only $2 n$. We remind the reader that the image space of a matrix $A$ consists of all vectors $x$ that can be written as $x=A y$ for some vector $y$; the dimension of this linear subspace is denoted by $\operatorname{rank} A$. The image space of $\Omega$, which has dimension $2 n=\operatorname{rank} \Omega$, is identical to the tangent space of the symplectic leaf at $x=0$. Vectors that are not contained in the image space of $\Omega$ correspond to an infinitesimal displacement of the system out of the symplectic leaf and are unphysical. We may thus restrict the matrices $\Omega$ and $M \Omega$ to the image space of $\Omega$. We denote these restricted matrices by $\langle\Omega\rangle$ and $\langle M \Omega\rangle$; that is, we define

$$
\langle\Omega\rangle \equiv F^{\top} \Omega F \quad \text { and } \quad\langle M \Omega\rangle \equiv F^{\top} M \Omega F,
$$

where $F$ is an $m \times 2 n$ matrix whose columns form an orthonormal basis of the image space of $\Omega$. Since the image space of $M \Omega$ is contained in the image space of $\Omega$, these restrictions are well defined and without loss. Notice that the matrix $\langle\Omega\rangle$ is invertible by definition. In this paper, we implicitly convert between vectors in $\mathbb{R}^{2 n}$ and vectors in the image space of $\Omega$ without writing $F$. It is unnecessary to explicitly construct $F$ in a computer code (see Sec. VII).

It can be shown (see the Appendix) that the $2 n \times 2 n$ matrix $\langle M \Omega\rangle$ is the Hessian matrix (the matrix of second-order partial derivatives) at $x=0$ of the restriction of the Hamiltonian $\mathcal{H}$ to the symplectic leaf (for a certain parametrization of the symplectic leaf). Therefore, if $x=0$ is a constrained local minimum of $\mathcal{H}$ on the symplectic leaf, the Hessian matrix $\langle M \Omega\rangle$ is guaranteed to be positive semidefinite. However, it may not be assumed (compare Ref. [31]) that $\langle M \Omega\rangle$ is also positive definite. To see this, consider the following simple

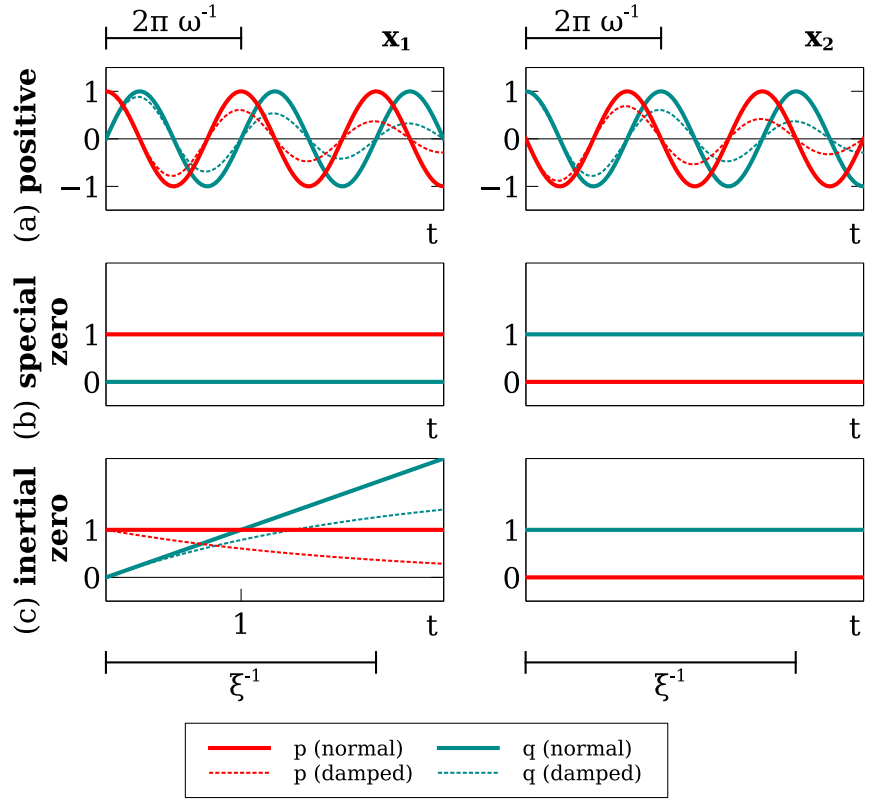

FIG. 1. (Color online) Fundamental solutions $x_{1}$ and $x_{2}$ of the linearized equation of motion (1) corresponding to the three types of normal modes of a Hamiltonian system: (a) positive (3), (b) special zero (5) and (c) inertial zero (7) modes. The dynamical variables $p$ and $q$ are the amplitudes of the vectors $u_{1}$ and $u_{2}$ respectively, as defined in Eq. (12). Dashed lines: effect of damping with the indicated decay time $\xi^{-1}$ (see Sec. V).

counterexamples with $m=2 n=2: \mathcal{H}(p, q)=0, \mathcal{H}(p, q)=$ $p^{2}$, and $\mathcal{H}(p, q)=p^{4}+q^{4}$ all have minima at $p=q=0$ but not positive-definite Hessians at that point.

If $\langle M \Omega\rangle$ is positive semidefinite, the normal modes of $M$ may be of three distinct types (see Appendix). We introduce the following names for these three types of modes.

(1) A positive normal mode of $M$ is a pair $\left(u_{1}, u_{2}\right)$ of vectors in the image space of $\Omega$ that satisfy

$$
M u_{1}=\omega u_{2}, \quad M u_{2}=-\omega u_{1},
$$

for some $\omega>0$. The corresponding fundamental solutions of the linearization of Eq. (1) are [see Fig. 1(a)]

$$
\begin{aligned}
& x_{1}(t)=\cos (\omega t) u_{1}+\sin (\omega t) u_{2}, \\
& x_{2}(t)=-\sin (\omega t) u_{1}+\cos (\omega t) u_{2} .
\end{aligned}
$$

Each positive normal mode corresponds to a pair of eigenvectors of $M$. The eigenvectors are $u_{1}-i u_{2}$ (eigenvalue $i \omega$ ) and $u_{1}+i u_{2}$ (eigenvalue $-i \omega$ ).

(2) A special zero normal mode is a pair $\left(u_{1}, u_{2}\right)$ of vectors in the image space of $\Omega$ that satisfy

$$
M u_{1}=0, \quad M u_{2}=0 .
$$

The corresponding fundamental solutions are [see Fig. 1(b)]

$$
x_{1}(t)=u_{1}, \quad x_{2}(t)=u_{2}
$$

(constant functions). A special zero normal mode also corresponds to a pair of linearly independent eigenvectors of $M\left(u_{1}\right.$ and $u_{2}$ ). 
(3) An inertial zero normal mode is a pair $\left(u_{1}, u_{2}\right)$ of vectors in the image space of $\Omega$ that satisfy

$$
M u_{1}=u_{2}, \quad M u_{2}=0 .
$$

The corresponding fundamental solutions are [see Fig. 1(c)]

$$
x_{1}(t)=u_{1}+t u_{2}, \quad x_{2}(t)=u_{2} .
$$

This type of mode results from a nondiagonalizable (defective) matrix $M$. Technically, an inertial zero mode corresponds to a Jordan block of size 2 in the Jordan normal form of $M$.

The nomenclature chosen for the three types of modes (positive, special, and inertial) is explained below. Notice that different types of modes may have different units: For an inertial zero normal mode, $\left\|u_{1}\right\| /\left\|u_{2}\right\|$ has units of time, while for a positive normal mode, $\left\|u_{1}\right\| /\left\|u_{2}\right\|$ is dimensionless. Since each mode contains two vectors, the total number of independent modes $n$ is one half of the dimension of the symplectic leaf. If $\langle M \Omega\rangle$ is positive definite, all normal modes are positive normal modes.

We may write the vectors that make up a normal mode as

$$
u_{1}=\Omega w_{1}, \quad u_{2}=\Omega w_{2},
$$

for certain vectors $w_{1}$ and $w_{2}$ in the image space of $\Omega$. Section IV presents an efficient procedure by which suitable vector pairs $w_{1}, w_{2}$ may be found. All normal modes can and should be chosen to satisfy the relations

$$
\begin{aligned}
& w_{1 k}^{\top} \Omega w_{2 l}=\delta_{k l}, \\
& w_{1 k}^{\top} \Omega w_{1 l}=w_{2 k}^{\top} \Omega w_{2 l}=0,
\end{aligned}
$$

where $k, l=1, \ldots, n$ index the modes. As a result, we may decompose an arbitrary vector $x$ in the image space of $\Omega$ in terms of the normal modes as

$$
x=\sum_{k=1}^{n}\left[-\left(w_{2 k}^{\top} x\right) u_{1 k}+\left(w_{1 k}^{\top} x\right) u_{2 k}\right] .
$$

Using the fundamental solutions (4), (6), and (8), such a decomposition immediately yields a solution of the initialvalue problem for Eq. (1) in the linear approximation.

Given a state vector

$$
x=\sum_{k=1}^{n}\left(p_{k} u_{1 k}+q_{k} u_{2 k}\right)+\mathcal{O}\left(p_{k}^{2}+q_{k}^{2}\right),
$$

the quadratic part of the Hamiltonian is given by

$$
\mathcal{H}=\sum_{k \text { pos. }} \frac{1}{2} \omega_{k}\left(p_{k}^{2}+q_{k}^{2}\right)+\sum_{k \text { def. }} \frac{1}{2} p_{k}^{2},
$$

where the first sum is taken over the positive normal modes and the second sum over the inertial zero normal modes. Special zero modes do not contribute to Eq. (13). The variables $p_{k}$ and $q_{k}$ in Eq. (12) are canonically conjugate momenta and coordinates (see the Appendix). Notice that for a given configuration $m=m_{0}+x$, the values of these momenta and coordinates can be determined, to first order, using Eq. (11). We find, in the linear limit, that for a special zero normal mode,

$$
\dot{p}_{k}=-\frac{\partial \mathcal{H}}{\partial q_{k}}=0, \quad \dot{q}_{k}=\frac{\partial \mathcal{H}}{\partial p_{k}}=0,
$$

while for an inertial zero normal mode,

$$
\dot{p}_{k}=-\frac{\partial \mathcal{H}}{\partial q_{k}}=0, \quad \dot{q}_{k}=\frac{\partial \mathcal{H}}{\partial p_{k}}=p_{k} .
$$

The latter type of dynamics (15) corresponds (after a suitable scaling of $p_{k}$ and $q_{k}$ ) to the dynamics of a free massive particle, which explains our choice of the name "inertial zero normal mode." The former type of dynamics (14) does not occur in conventional Newtonian systems such as systems of coupled oscillators (see the Appendix), whence the name "special zero normal mode."

In practice, zero modes typically originate from a broken continuous symmetry and can thus be seen as Goldstone modes. For example, $q_{k}$ might represent the position of some topological defect; if the system is translationally invariant, the Hamiltonian must be independent of $q_{k}$. The main difference between the two types of zero modes is the number of broken continuous symmetries from which they arise. For an inertial zero mode $k$, the Hamiltonian (13) is, to second order, independent of $q_{k}$ (but not of $p_{k}$ ). This suggests (but of course does not guarantee) that the system is invariant under arbitrary changes of $q_{k}$. A special zero mode $k$ is special in the sense that the Hamiltonian is to second order independent of both $q_{k}$ and $p_{k}$. This normally means that two broken continuous symmetries coexist. The relation among the type of zero mode, the number of broken continuous symmetries, and the dispersion relation of soft modes is discussed in more detail in Sec. VIII D.

\section{CONSERVATIVE SPIN SYSTEMS}

The conservative dynamics of a spin system is described by the LLG equation without damping,

$$
\dot{\mathbf{m}}_{i}=\tilde{\gamma} \mathbf{m}_{i} \times \nabla_{\mathbf{m}_{i}} \mathcal{H},
$$

where $\mathbf{m}_{i} \in \mathbb{R}^{3}$ represents the magnetic moment with position index $i=1, \ldots, n, \mathcal{H}$ is the Hamiltonian, and $\tilde{\gamma}$ is a physical constant. Notice that the magnitude $\left\|\mathbf{m}_{i}\right\|$ of each magnetic moment is constant in time. These magnitudes are fixed by the physics of the system. Equation (16) is equivalent to

$$
\dot{m}_{i \alpha}=\left\{m_{i \alpha}, \mathcal{H}\right\},
$$

the generalized form of Hamilton's equations applied to the (time-invariant) variables $m_{i \alpha}$, for the Poisson bracket

$$
\left\{m_{i \alpha}, m_{j \beta}\right\}= \begin{cases}-\tilde{\gamma} \varepsilon_{\alpha \beta \gamma} m_{i \gamma} & \text { for } i=j, \\ 0 & \text { for } i \neq j,\end{cases}
$$

where Greek indices represent Cartesian coordinates $x, y, z$ and $\varepsilon_{\alpha \beta \gamma}$ is the Levi-Civita symbol. Thus, the dynamics of the conservative spin system is Hamiltonian.

For convenience, we write Eq. (16) as

$$
\dot{m}=\tilde{\gamma}[m, \nabla \mathcal{H}] .
$$

The variable $m \in \mathbb{R}^{3 n}$ can be seen as a compound vector that assigns to every position $i=1, \ldots, n$ a vector $\mathbf{m}_{i} \in \mathbb{R}^{3}$. The square brackets in Eq. (19) denote an elementwise cross product: Given $x, y \in \mathbb{R}^{3 n}$, we define $z=[x, y] \in \mathbb{R}^{3 n}$ such that $\mathbf{z}_{i}=\mathbf{x}_{i} \times \mathbf{y}_{i}$ for each position $i$. In other words, it is just the ordinary cross product (vector product) performed 
$n$ times. For small deviations $x=m-m_{0} \in \mathbb{R}^{3 n}$ from some fixed configuration $m_{0}$, Eq. (19) becomes

$$
\dot{x}=-\tilde{\gamma}\left[m_{0}, h\right]+M x+\mathcal{O}\left(\|x\|^{2}\right),
$$

where $h_{(i \alpha)}=-\partial \mathcal{H} /\left.\partial m_{i \alpha}\right|_{m=m_{0}}$ is the effective field at $m_{0}$. The matrix $M$ is given by

$$
M x=\tilde{\gamma}\left[m_{0}, A x\right]+\tilde{\gamma}[h, x],
$$

where $A_{(i \alpha)(j \beta)}=\partial^{2} \mathcal{H} /\left.\left(\partial m_{i \alpha} \partial m_{j \beta}\right)\right|_{m=m_{0}}$ is the $3 n \times 3 n$ Hessian matrix of $\mathcal{H}$ at $m_{0}$. To be explicit, let us mention that the elements of $M$ are given by

$M_{(i \alpha)(j \beta)}= \begin{cases}\tilde{\gamma} \varepsilon_{\alpha \gamma \delta}\left(m_{0}\right)_{i \gamma} A_{(i \delta)(j \beta)}+\tilde{\gamma} \varepsilon_{\alpha \gamma \beta} h_{i \gamma} & \text { for } i=j, \\ \tilde{\gamma} \varepsilon_{\alpha \gamma \delta}\left(m_{0}\right)_{i \gamma} A_{(i \delta)(j \beta)} & \text { for } i \neq j .\end{cases}$

(Summation is implied for repeated Greek indices but not for repeated Roman indices.) Since we work in Cartesian coordinates, $A$ is typically of a relatively simple form. Indeed, many micromagnetic models use a Hamiltonian that is quadratic in the Cartesian coordinates, in which case $A$ does not depend on $m_{0}$.

We assume that $m_{0}$ is an equilibrium configuration, $\left[m_{0}, h\right]=0$. As a result, Eq. (20) is of the form (1). The matrix $\Omega$ (2) is given by

$$
\begin{aligned}
\Omega_{(i \alpha)(j \beta)} & =-\left.\left\{m_{i \alpha}, m_{j \beta}\right\}\right|_{m=m_{0}} \\
& = \begin{cases}\tilde{\gamma} \varepsilon_{\alpha \beta \gamma}\left(m_{0}\right)_{i \gamma} & \text { for } i=j, \\
0 & \text { for } i \neq j,\end{cases}
\end{aligned}
$$

or, equivalently,

$$
\Omega x=-\tilde{\gamma}\left[m_{0}, x\right] .
$$

The $2 n$-dimensional image space of $\Omega$ consists of vectors $x \in$ $\mathbb{R}^{3 n}$ for which the displacement $\mathbf{x}_{i} \in \mathbb{R}^{3}$ is orthogonal at each position $i$ to the equilibrium direction $\mathbf{m}_{0 i}$. Notice also that the equilibrium effective field $\mathbf{h}_{i}$ must be parallel at each position $i$ to the equilibrium direction $\mathbf{m}_{0 i}$. Combining Eqs. (21) and (24), the matrix $M \Omega$, which is symmetric (see Sec. II), is given by

$$
\begin{aligned}
M \Omega x & =-\tilde{\gamma}^{2}\left(\left[m_{0}, A\left[m_{0}, x\right]\right]+\left[h,\left[m_{0}, x\right]\right]\right) \\
& =\left(\Omega^{\top} A \Omega+\tilde{\gamma}[h, \cdot] \Omega\right) x .
\end{aligned}
$$

The second term, which contains $h$, originates from the fact that the Hessian matrix $A$ is calculated in Cartesian coordinates, while the symplectic leaf (a product of $n$ spheres) is curved.

\section{REDUCTION TO THE HDGEP}

In this section, we present a method for the solution of the normal-mode problem of a general Hamiltonian system near a local minimum of the Hamiltonian. This includes the normal-mode problem of the conservative spin system as a special case. We show that the normal-mode problem can be reduced to the HDGEP, in which form it can be efficiently solved (see Sec. VII). Our method calculates both the positive modes and any zero modes of the system. If zero modes are present, the method detects these and automatically determines their types (special or inertial).

The conservative spin system differs from an important subclass of Hamiltonian systems, which includes systems of coupled point masses, for which the normal-mode problem can be written as a symmetric definite generalized eigenvalue problem (SDGEP) in an obvious way (see the Appendix). Such Hamiltonian systems are defined on a natural set of canonical momenta and coordinates. In terms of these, the Hamiltonian is of the form $\mathcal{H}\left(\left\{p_{i}\right\},\left\{q_{i}\right\}\right)=\mathcal{T}\left(\left\{p_{i}\right\}\right)+\mathcal{V}\left(\left\{q_{i}\right\}\right)$, where the potential-energy term $\mathcal{V}$ depends only on the coordinates $q_{i}$, while the kinetic-energy term $\mathcal{T}$ is a positive-definite quadratic function depending only on the momenta $p_{i}$ [typically, $\mathcal{T}=$ $\left.\sum_{i} p_{i}^{2} /\left(2 m_{i}\right)\right]$. The spin system is not of this special form. All that is given is the Poisson bracket (18) and the Hamiltonian $\mathcal{H}\left(\left\{\mathbf{m}_{i}\right\}\right)$ as a function of the magnetic moments $\mathbf{m}_{i}$. Even though it is possible to construct canonical momenta and coordinates [47] for this system, an a priori separation of kinetic energy and potential energy is not normally known.

We first consider the case that $\langle M \Omega\rangle$ is positive definite (no zero modes). Later in this section we treat the general case where $\langle M \Omega\rangle$ is positive semidefinite. This generalization is essential for spin systems such as those discussed in Secs. VIII B-VIII E.

We remind the reader that an HDGEP has the general form

$$
D x=\lambda S x,
$$

where $D$ is Hermitian and $S$ is Hermitian and positive definite, which requirements guarantee that all eigenvalues $\lambda_{i}$ are real. The usual Hermitian eigenvalue problem is a special case of the HDGEP (set $S=I$ ). If $D$ and $S$ are real matrices, so that $D$ and $S$ are symmetric, we use the abbreviation SDGEP. The eigenvectors $x_{i}$ of a HDGEP may be chosen to satisfy $x_{i}^{\dagger} S x_{j}=$ $\delta_{i j}$, a generalized orthonormality relation. Alternatively, we may choose to normalize the eigenvectors $x_{i}$ in such a way that

$$
x_{i}^{\dagger} D x_{j}= \pm \delta_{i j}
$$

provided that $D$ is invertible, in which case the eigenvalues $\lambda_{i}$ are nonzero.

Let us represent a positive normal mode (3) as a single vector

$$
w=w_{1}+i w_{2} \in \mathbb{C}^{2 n},
$$

where $w_{1}$ and $w_{2}$ are such that

$$
u_{1}=\langle\Omega\rangle w_{1} \quad \text { and } \quad u_{2}=\langle\Omega\rangle w_{2} .
$$

It is easy to see that in this notation, a solution of the generalized eigenvalue problem,

$$
\langle M \Omega\rangle w=-i \omega\langle\Omega\rangle w,
$$

with $\omega>0$ is also a solution of Eq. (3) (after conversion of the vectors in $\mathbb{R}^{2 n}$ to vectors in the image space of $\Omega$ ): Take real and imaginary parts. If we assume that $\langle M \Omega\rangle$ is positive definite, Eq. (29) is a HDGEP (26) with $D=-i\langle\Omega\rangle, S=\langle M \Omega\rangle$ and $\lambda=\omega^{-1}$, since $\Omega$ is antisymmetric and $M \Omega$ is symmetric (see Sec. II). The HDGEP form (29) makes the problem suitable for efficient numerical computation. Notice that $\lambda \neq$ 0 , since $\langle\Omega\rangle$ is invertible. Notice also that each positive normal mode gives rise to two independent solutions of Eq. (29): If $w=w_{1}+i w_{2}$ is an eigenvector with eigenvalue $\omega>0$, then $w^{*}=w_{1}-i w_{2}$ is an eigenvector with eigenvalue $-\omega$. By Eq. (27), we may normalize the eigenvectors so that they 
satisfy

$$
\begin{aligned}
& \left(w_{1 k}+i w_{2 k}\right)^{\dagger}(-i\langle\Omega\rangle)\left(w_{1 l}+i w_{2 l}\right)=2 \delta_{k l} \\
& \left(w_{1 k}-i w_{2 k}\right)^{\dagger}(-i\langle\Omega\rangle)\left(w_{1 l}+i w_{2 l}\right)=0
\end{aligned}
$$

which together are equivalent to Eqs. (10a) and (10b).

Equation (29) can be seen as a generalization of Eqs. (27)-(30) in Ref. [31], which were given for the normalmode problem of the conservative spin system, to a general Hamiltonian system. Our formulation has the additional advantage that it does not require the use of spherical coordinates. By itself, the method only works if $\langle M \Omega\rangle$ is positive definite. If $\langle M \Omega\rangle$ is merely positive semidefinite, Eq. (29) is no longer a HDGEP. Zero normal modes may appear and the matrix $M$ is not even guaranteed to be diagonalizable. These zero modes have important consequences for the dynamics of, for example, domain walls or Skyrmions in magnetic systems (see Secs. VIII B-VIIIE). We present here a robust scheme that also works in this more general case. Thus, our method can solve the normal-mode problem of any Hamiltonian system linearized at a local minimum of the Hamiltonian.

The main idea of our approach is that we first find the special and inertial zero normal modes and then exclude them from the problem. The algorithm consists of the steps outlined below. The only two "large" ( $2 n$-dimensional) problems in this procedure are steps (1) and (4). In step (1), we need to find the lowest eigenvalues and corresponding eigenvectors of a symmetric matrix. In step (4), we need to solve a symmetric linear system. Both subproblems can be efficiently solved using iterative methods. How this may be done is discussed in more detail in Sec. VII. The diagonalizations in steps (2) and (5) concern small matrices and can be performed using standard routines.

(1) Sequentially find the eigenvectors $y_{1}, y_{2}, \ldots \in \mathbb{R}^{2 n}$ of $\langle M \Omega\rangle$ that correspond to the lowest eigenvalues (see Sec. VII). Stop when an eigenvector appears with an eigenvalue that is larger than zero (by a certain small tolerance). Notice that this is an ordinary (nongeneralized) symmetric eigenvalue problem, so that the fact that $\langle M \Omega\rangle$ is not necessarily positive definite is not a problem. By positive semidefiniteness of $\langle M \Omega\rangle$, all eigenvalues are greater than or equal to zero. Suppose that there are $s$ eigenvectors with eigenvalue zero. Then $y_{1}, \ldots, y_{s}$ form a basis of the null space of $\langle M \Omega\rangle$. In most cases, $s$ will be a small number. Remember that, thanks to the restriction of $M \Omega$ to $\langle M \Omega\rangle$, we have already excluded all null vectors of $M \Omega$ that are also null vectors of $\Omega$ and thus correspond to a displacement of the system out of the symplectic leaf.

(2) Define the $s \times s$ matrix $[\Omega]_{i j}=y_{i}^{\top}\langle\Omega\rangle y_{j}$. Since $[\Omega]_{i j}$ is antisymmetric, $i[\Omega]_{i j}$ is Hermitian and can be diagonalized by a standard routine for Hermitian matrices, which guarantees that the eigenvectors are orthonormal. Let $s_{\mathrm{d}}$ be the number of independent eigenvectors $B_{i(k)}$ of $[\Omega]_{i j}$ with eigenvalue zero (up to a small tolerance). We have $\sum_{j=1}^{s}[\Omega]_{i j} B_{j(k)}=$ 0 for $k=1, \ldots, s_{\mathrm{d}}$. We may take these eigenvectors $B_{i(k)}$ to be real. The remaining non-null eigenvectors come in $s_{\mathrm{o}}$ pairs. Let $C_{i(l)}+i D_{i(l)}$ be an eigenvector of $[\Omega]_{i j}$ with eigenvalue $i \lambda_{(l)}$, where $\lambda_{(l)}>0$ and $C_{i(l)}$ and $D_{i(l)}$ are real. We have $\sum_{j=1}^{s}[\Omega]_{i j}\left(C_{j(l)}+i D_{j(l)}\right)=i \lambda_{(l)}\left(C_{i(l)}+i D_{i(l)}\right)$ for $l=$ $1, \ldots, s_{0}$. Then $C_{i(l)}-i D_{i(l)}$ is an eigenvector with eigenvalue $-i \lambda_{(l)}$. The total number of eigenvectors is $s=s_{\mathrm{d}}+2 s_{\mathrm{o}}$.
(3) Construct the vectors $c_{l}=\sum_{i=1}^{s} C_{i(l)} y_{i}$ and $d_{l}=$ $\sum_{i=1}^{s} D_{i(l)} y_{i}$ for $l=1, \ldots, s_{\mathrm{o}}$ and $\bar{b}_{k}=\sum_{i=1}^{s} B_{i(k)} y_{i}$ for $k=$ $1, \ldots, s_{\mathrm{d}}$. Notice that we have $c_{l}^{\top}\langle\Omega\rangle d_{l^{\prime}}=0$ for $l \neq l^{\prime}$ and $c_{l}^{\top}\langle\Omega\rangle d_{l}>0$. Moreover, for all $l, l^{\prime}, k, k^{\prime}$ we have $c_{l}^{\top}\langle\Omega\rangle c_{l^{\prime}}=$ $d_{l}^{\top}\langle\Omega\rangle d_{l^{\prime}}=0, c_{l}^{\top}\langle\Omega\rangle \bar{b}_{k}=d_{l}^{\top}\langle\Omega\rangle \bar{b}_{k}=0$, and $\bar{b}_{k}^{\top}\langle\Omega\rangle \bar{b}_{k^{\prime}}=0$.

(4) For each $k=1, \ldots, s_{\mathrm{d}}$, find a vector $\bar{a}_{k}$ such that $\langle M \Omega\rangle \bar{a}_{k}=\langle\Omega\rangle \bar{b}_{k}$ (see Sec. VII). We know that such a vector exists, since by construction $\langle\Omega\rangle \bar{b}_{k}$ lies in the orthogonal complement of the null space of $\langle M \Omega\rangle$, a symmetric matrix, and hence in the image space of $\langle M \Omega\rangle$. Although this vector $\bar{a}_{k}$ is not uniquely defined, there is a unique solution $\bar{a}_{k}$ that lies in the image space of $\langle M \Omega\rangle$, which is the solution that is obtained by the method given in Sec. VII.

(5) Define the symmetric $s_{\mathrm{d}} \times s_{\mathrm{d}}$ matrix $[\mathrm{M} \Omega]_{k k^{\prime}}=$ $\bar{a}_{k}^{\top}\langle M \Omega\rangle \bar{a}_{k^{\prime}}$ and diagonalize it using a standard routine for symmetric matrices. Let the orthonormal eigenvectors be $G_{i(k)}$. We have $\sum_{j=1}^{s_{\mathrm{d}}}[\mathrm{M} \Omega]_{i j} G_{j(k)}=\mu_{(k)} G_{i(k)}$ with $\mu_{(k)}>0$ for $k=1, \ldots, s_{\mathrm{d}}$. The eigenvalues $\mu_{(k)}$ are positive, since $\langle M \Omega\rangle$ is positive semidefinite and the vectors $\bar{a}_{k}$ are independent vectors in the image space of $\langle M \Omega\rangle$.

(6) Construct the vectors $b_{k}=\sum_{i=1}^{s_{\mathrm{d}}} G_{i(k)} \bar{b}_{i}$ and $\breve{a}_{k}=$ $\sum_{i=1}^{s_{\mathrm{d}}} G_{i(k)} \bar{a}_{i}$ for $k=1, \ldots, s_{\mathrm{d}}$. Since $\breve{a}_{k}^{\top}\langle\Omega\rangle b_{k^{\prime}}=\breve{a}_{k}^{\top}\langle M \Omega\rangle \breve{a}_{k^{\prime}}$, we have $\breve{a}_{k}^{\top}\langle\Omega\rangle b_{k^{\prime}}=0$ for $k \neq k^{\prime}$ and $\breve{a}_{k}^{\top}\langle\Omega\rangle b_{k}>0$.

(7) Redefine $\breve{a}_{k}$ as $\breve{a}_{k} / \sqrt{\alpha_{k}}, \quad b_{k}$ as $b_{k} / \sqrt{\alpha_{k}}, \quad c_{l}$ as $c_{l} / \sqrt{\beta_{l}}$, and $d_{l}$ as $d_{l} / \sqrt{\beta_{l}}$, where $\alpha_{k}=\breve{a}_{k}^{\top}\langle\Omega\rangle b_{k}=\mu_{(k)}$ and $\beta_{l}=c_{l}^{\top}\langle\Omega\rangle d_{l}=\lambda_{(l)} / 2$. This normalizes the modes so that $\breve{a}_{k}^{\top}\langle\Omega\rangle b_{k}=1$ for each $k$ and $c_{l}^{\top}\langle\Omega\rangle d_{l}=1$ for each $l$.

(8) Set $\hat{a}_{k}=\breve{a}_{k}-\sum_{l=1}^{s_{\mathrm{o}}}\left(c_{l}^{\top}\langle\Omega\rangle \breve{a}_{k}\right) d_{l}+\sum_{l=1}^{s_{\mathrm{o}}}\left(d_{l}^{\top}\langle\Omega\rangle \breve{a}_{k}\right) c_{l}$. We have $c_{l}^{\top}\langle\Omega\rangle \hat{a}_{k}=d_{l}^{\top}\langle\Omega\rangle \hat{a}_{k}=0$ for all $l, k$.

(9) Set $a_{k}=\hat{a}_{k}-\sum_{k^{\prime}=1}^{s_{\mathrm{d}}} \frac{1}{2}\left(\hat{a}_{k^{\prime}}^{\top}\langle\Omega\rangle \hat{a}_{k}\right) b_{k^{\prime}}$. We have $a_{k}^{\top}\langle\Omega\rangle a_{k^{\prime}}=0$ for all $k, k^{\prime}$.

(10) The pairs $\left(u_{1}, u_{2}\right)=\left(\Omega a_{k}, \Omega b_{k}\right)$ are the inertial zero normal modes (7). The pairs $\left(u_{1}, u_{2}\right)=\left(\Omega c_{l}, \Omega d_{l}\right)$ are the special zero normal modes (5). All zero normal modes now satisfy the relations (10a) and (10b).

Let us define the zero normal modes, of which there are $s_{\mathrm{d}}+s_{\mathrm{O}}$, as the first modes in the list of all modes: set $w_{1 i}=a_{i}, w_{2 i}=b_{i}$ for $i=1, \ldots, s_{\mathrm{d}}$ and $w_{1\left(s_{\mathrm{d}}+i\right)}=c_{i}$, $w_{2\left(s_{\mathrm{d}}+i\right)}=d_{i}$ for $i=1, \ldots, s_{\mathrm{O}}$. All normal modes must satisfy the relations (30a) and (30b), which are equivalent to Eqs. (10a) and (10b). Once the zero normal modes have been obtained, we may thus restrict the generalized eigenvalue problem (29) to trial vectors $w$ that satisfy

$$
\begin{aligned}
& \left(w_{1 i}+i w_{2 i}\right)^{\dagger}\langle\Omega\rangle w=0, \\
& \left(w_{1 i}-i w_{2 i}\right)^{\dagger}\langle\Omega\rangle w=0,
\end{aligned}
$$

for all zero normal modes $i=1, \ldots, s_{\mathrm{d}}+s_{\mathrm{o}}$. These constraints can be implemented in the iterative HDGEP solver in a very natural way (see Sec. VII). On this subspace, Eq. (29) constitutes an HDGEP, so we can efficiently find the remaining modes $i=s_{\mathrm{d}}+s_{\mathrm{o}}+1, \ldots, n$.

\section{DAMPED SPIN SYSTEMS}

We have seen that the magnetic normal modes of a conservative spin system, which is Hamiltonian, can be obtained by solving a HDGEP. However, typical magnetic systems can be modeled more realistically using the LLG 
equation [7] with a nonzero damping parameter $\eta>0$,

$$
\dot{\mathbf{m}}_{i}=-\tilde{\gamma} \mathbf{m}_{i} \times\left(-\nabla_{\mathbf{m}_{i}} \mathcal{H}-\eta \dot{\mathbf{m}}_{i}\right)
$$

[compare Eq. (16)]. Note that some texts write the LLG equation with damping (32) in a somewhat different, explicit form [6,7]. The damping term affects the magnetic normal modes and the eigenfrequencies $\omega$, which now acquire an imaginary part $[48,49]$. Our method for the magnetic normalmode problem can be used even in this nonconservative case if we treat the damping term of the LLG equation as a perturbation. We are justified in doing so, since $\eta$ is often small ( $\eta \ll 1 / \tilde{\gamma} m_{\mathrm{S}}$, where $m_{\mathrm{S}}$ is the typical magnitude $\left\|\mathbf{m}_{i}\right\|$ of the spins). In this way, we avoid the need for the solution of non-Hermitian eigenvalue problems [50] and describe the effect of damping directly in terms of the conservative normal modes, which correspond to canonical coordinates.

In this section, we derive expressions for the damped modes in first-order perturbation theory. In particular, we obtain very simple and elegant first-order expressions (40) and (43) for the decay rate of the amplitude of a mode under damping. Decay rates of modes are especially important as they determine the widths of the corresponding peaks in dynamic magnetic susceptibility functions (see Fig. 6), which can be measured. In contrast to previous applications of the perturbative method [51], our expressions provide first-order corrections to the mode vectors $u_{1}, u_{2}$ (even for degenerate normal modes) and also cover systems with special or inertial zero normal modes.

Again considering the deviation $x=m-m_{0} \in \mathbb{R}^{3 n}$ from a fixed equilibrium configuration $m_{0}$ in Cartesian coordinates (see Sec. III), the LLG equation with damping (32) becomes, using that $\dot{x}=\mathcal{O}(\|x\|)$,

$$
\dot{x}=M x-\eta \Omega \dot{x}+\mathcal{O}\left(\|x\|^{2}\right),
$$

with $M$ and $\Omega$ as defined in Eqs. (21) and (24). We can write this in explicit form as $\dot{x}=M^{\prime} x+\mathcal{O}\left(\|x\|^{2}\right)$, where

$$
M^{\prime}=\left(I_{3 n}+\eta \Omega\right)^{-1} M=\left(I_{3 n}+\eta^{2} \Omega^{\top} \Omega\right)^{-1}(M-\eta \Omega M) .
$$

We see that to first order in $\eta$, the matrix $M^{\prime} \Omega$ results from perturbation of $M \Omega$ by a term $-\eta \Omega M \Omega$. Since the LLG equation with damping (32) respects the constraint that the magnitude $\left\|\mathbf{m}_{i}\right\|$ of each magnetic moment be constant, we may still assume that the physically relevant vectors $x \in \mathbb{R}^{3 n}$ lie in the $2 n$-dimensional image space of $\Omega$.

The presence of (a not-too-large amount of) damping modifies the three types of normal modes as follows. We use primes for the modes of the damped system.
(1) A postive normal mode (3) becomes a damped mode of the form

$$
M^{\prime} u_{1}^{\prime}=\omega^{\prime} u_{2}^{\prime}-\xi^{\prime} u_{1}^{\prime}, \quad M^{\prime} u_{2}^{\prime}=-\omega^{\prime} u_{1}^{\prime}-\xi^{\prime} u_{2}^{\prime} .
$$

The fundamental solutions that correspond to a damped positive mode (35) are

$$
\begin{aligned}
& x_{1}(t)=e^{-\xi^{\prime} t}\left[\cos \left(\omega^{\prime} t\right) u_{1}^{\prime}+\sin \left(\omega^{\prime} t\right) u_{2}^{\prime}\right], \\
& x_{2}(t)=e^{-\xi^{\prime} t}\left[-\sin \left(\omega^{\prime} t\right) u_{1}^{\prime}+\cos \left(\omega^{\prime} t\right) u_{2}^{\prime}\right] .
\end{aligned}
$$

(2) A special zero normal mode (5) remains unchanged in the presence of damping.

(3) An inertial zero normal mode (7) becomes a damped mode of the form

$$
M^{\prime} u_{1}^{\prime}=u_{2}-\xi^{\prime} u_{1}^{\prime}, \quad M^{\prime} u_{2}=0 .
$$

The corresponding fundamental solutions are

$$
x_{1}(t)=e^{-\xi^{\prime} t} u_{1}^{\prime}+\left[\left(1-e^{-\xi^{\prime} t}\right) / \xi^{\prime}\right] u_{2}, \quad x_{2}(t)=u_{2} .
$$

Notice that the $u_{2}$ part of an inertial normal mode remains unchanged in the presence of damping.

Equation (35) is equivalent to Eq. (29) if we replace $M$ in Eq. (29) with $M^{\prime}$ and $\omega$ with $\omega^{\prime}-i \xi^{\prime}$; it is in this sense that the frequency of a damped positive mode acquires an imaginary part. Notice that with damping, Eq. (29) is no longer a HDGEP. As a result, the damped modes do not necessarily satisfy the relations (10a) and (10b).

In first-order perturbation theory, we write a damped positive mode (35) as

$$
\begin{aligned}
\omega_{k}^{\prime} & =\omega_{k}+\mathcal{O}\left(\eta^{2}\right), \\
\xi_{k}^{\prime} & =\eta \xi_{k}^{(1)}+\mathcal{O}\left(\eta^{2}\right), \\
u_{1 k}^{\prime} & =u_{1 k}+\eta u_{1 k}^{(1)}+\mathcal{O}\left(\eta^{2}\right), \\
u_{2 k}^{\prime} & =u_{2 k}+\eta u_{2 k}^{(1)}+\mathcal{O}\left(\eta^{2}\right),
\end{aligned}
$$

where $k$ is the mode index and $u_{1 k}, u_{2 k}$ and $\omega_{k}$ are the unperturbed normal mode and frequency, respectively. We assume that the vectors $w_{1}, w_{2}$ of all unperturbed normal modes (9) satisfy the relations (10a) and (10b). Moreover, we assume that if any of the unperturbed normal modes are degenerate, they satisfy certain additional conditions (stated below). Using these assumptions and the definitions (3), (5), and (7), it can be derived, by a rather lengthy calculation, that the first-order corrections to a positive mode $k$ are given by

$$
\begin{aligned}
\xi_{k}^{(1)}= & \frac{1}{2} \omega_{k}\left(u_{1 k}^{\top} u_{1 k}+u_{2 k}^{\top} u_{2 k}\right)=\frac{1}{2} \omega_{k}\left(\left\|u_{1 k}\right\|^{2}+\left\|u_{2 k}\right\|^{2}\right), \\
u_{1 k}^{(1)}= & \sum_{l\left(\omega_{l}=\omega_{k}\right)} \frac{1}{4}\left[-\left(u_{1 l}^{\top} u_{2 k}+u_{2 l}^{\top} u_{1 k}\right) u_{1 l}+\left(u_{1 l}^{\top} u_{1 k}-u_{2 l}^{\top} u_{2 k}\right) u_{2 l}\right] \\
& +\sum_{l\left(\omega_{l} \neq \omega_{k}\right)} \frac{1}{2} \omega_{k}\left[\left(\frac{u_{1 l}^{\top} u_{2 k}-u_{2 l}^{\top} u_{1 k}}{\omega_{k}-\omega_{l}}-\frac{u_{1 l}^{\top} u_{2 k}+u_{2 l}^{\top} u_{1 k}}{\omega_{k}+\omega_{l}}\right) u_{1 l}+\left(\frac{u_{1 l}^{\top} u_{1 k}+u_{2 l}^{\top} u_{2 k}}{\omega_{k}-\omega_{l}}+\frac{u_{1 l}^{\top} u_{1 k}-u_{2 l}^{\top} u_{2 k}}{\omega_{k}+\omega_{l}}\right) u_{2 l}\right] \\
& +\sum_{l \text { ord. }}\left[-\left(u_{2 l}^{\top} u_{1 k}\right) u_{1 l}+\left(u_{1 l}^{\top} u_{1 k}\right) u_{2 l}\right]+\sum_{l \text { def. }}\left[-\left(u_{2 l}^{\top} u_{1 k}\right) u_{1 l}+\left(u_{1 l}^{\top} u_{1 k}+\omega_{k}^{-1} u_{2 l}^{\top} u_{2 k}\right) u_{2 l}\right],
\end{aligned}
$$




$$
\begin{aligned}
u_{2 k}^{(1)}= & \sum_{l\left(\omega_{l}=\omega_{k}\right)} \frac{1}{4}\left[\left(u_{1 l}^{\top} u_{1 k}-u_{2 l}^{\top} u_{2 k}\right) u_{1 l}+\left(u_{1 l}^{\top} u_{2 k}+u_{2 l}^{\top} u_{1 k}\right) u_{2 l}\right] \\
& +\sum_{l\left(\omega_{l} \neq \omega_{k}\right)} \frac{1}{2} \omega_{k}\left[\left(-\frac{u_{1 l}^{\top} u_{1 k}+u_{2 l}^{\top} u_{2 k}}{\omega_{k}-\omega_{l}}+\frac{u_{1 l}^{\top} u_{1 k}-u_{2 l}^{\top} u_{2 k}}{\omega_{k}+\omega_{l}}\right) u_{1 l}+\left(\frac{u_{1 l}^{\top} u_{2 k}-u_{2 l}^{\top} u_{1 k}}{\omega_{k}-\omega_{l}}+\frac{u_{1 l}^{\top} u_{2 k}+u_{2 l}^{\top} u_{1 k}}{\omega_{k}+\omega_{l}}\right) u_{2 l}\right] \\
& +\sum_{l \text { ord. }}\left[-\left(u_{2 l}^{\top} u_{2 k}\right) u_{1 l}+\left(u_{1 l}^{\top} u_{2 k}\right) u_{2 l}\right]+\sum_{l \text { def. }}\left[-\left(u_{2 l}^{\top} u_{2 k}\right) u_{1 l}+\left(u_{1 l}^{\top} u_{2 k}-\omega_{k}^{-1} u_{2 l}^{\top} u_{1 k}\right) u_{2 l}\right],
\end{aligned}
$$

where the first sum in Eq. (41a) or (41b) is over any modes $l$ that are degenerate with the positive normal mode $k$, plus $k$ itself; the second sum is over all other positive normal modes; the third sum is over the special zero normal modes; and the fourth sum is over the inertial zero normal modes. For the damped inertial zero mode (37), we have

$$
\begin{aligned}
\xi_{k}^{\prime} & =\eta \xi_{k}^{(1)}+\mathcal{O}\left(\eta^{2}\right), \\
u_{1 k}^{\prime} & =u_{1 k}+\eta u_{1 k}^{(1)}+\mathcal{O}\left(\eta^{2}\right) .
\end{aligned}
$$

The first-order corrections are given by

$$
\begin{gathered}
\xi_{k}^{(1)}=u_{2 k}^{\top} u_{2 k}=\left\|u_{2 k}\right\|^{2}, \\
u_{1 k}^{(1)}=\sum_{l \text { pos. }}-\omega_{l}^{-1}\left[\left(u_{1 l}^{\top} u_{2 k}\right) u_{1 l}+\left(u_{2 l}^{\top} u_{2 k}\right) u_{2 l}\right] \\
+\sum_{l \text { def. }}-\left(u_{1 l}^{\top} u_{2 k}\right) u_{1 l},
\end{gathered}
$$

where the first sum in Eq. (44) is over all positive normal modes and the second sum is over all inertial zero normal modes. We see that in both cases (40) and (43), $\xi^{(1)}$ is guaranteed to be positive: for a positive damping parameter $\eta$, amplitudes of modes decrease in time. Notice that the frequency $\omega^{\prime}$ of a damped positive mode is constant to first order in $\eta$; however, there will be a second-order correction (normally negative).

If all magnetic moments in the equilibrium configuration $m_{0}$ have the same magnitude $\left\|\mathbf{m}_{0 i}\right\|=m_{\mathrm{S}}$, we have $\left\langle\Omega^{\top} \Omega\right\rangle=$ $\tilde{\gamma}^{2} m_{\mathrm{S}}^{2} I_{2 n}$, and Eq. (34) becomes

$$
M^{\prime} \Omega=\frac{1}{1+\left(\eta \tilde{\gamma} m_{\mathrm{S}}\right)^{2}}(M \Omega-\eta \Omega M \Omega) .
$$

We can then often further reduce the residual error in the damped positive modes, which is of second order in $\eta$, simply by dividing the $\omega^{\prime}$ and $\xi^{\prime}$ as obtained to first order by $1+$ $\left(\eta \tilde{\gamma} m_{\mathrm{S}}\right)^{2}$. For a damped inertial zero mode, divide the value $\xi^{\prime}$ by $1+\left(\eta \tilde{\gamma} m_{\mathrm{S}}\right)^{2}$ and multiply the vector $u_{1}^{\prime}$ by the same factor. These corrections do not eliminate the error of second order completely, but are very easy to implement.

If there are several distinct positive normal modes with the same frequency $\omega$, or if the dimension $s$ of the null space of $\langle M \Omega\rangle$ is larger than one, the normal-mode problem is degenerate. The damping perturbation may lift this degeneracy. For the correctness of the expressions for the first-order corrections it is essential to choose the degenerate unperturbed normal modes in such a way that the perturbation does not mix them. We amend the procedure of Sec. IV as follows. Given any symmetric and positive-definite matrix $A$, we may choose the null-space vectors $y_{1}, \ldots, y_{s}$ in step (1) of Sec. IV in such a way that they satisfy $y_{i}^{\top} A y_{j}=\delta_{i j}$. For the spin system with damping, we must use $A=\left\langle\Omega^{\top} \Omega\right\rangle$. The rest of the algorithm then automatically ensures that the vectors $b_{k}, c_{l}$, and $d_{l}$ [see step (10)] of the zero normal modes satisfy

$$
\begin{aligned}
& b_{k}^{\top} A b_{k^{\prime}}=0 \quad\left(\text { for } k \neq k^{\prime}\right), \\
& c_{l}^{\top} A c_{l^{\prime}}=d_{l}^{\top} A d_{l^{\prime}}=0 \quad\left(\text { for } l \neq l^{\prime}\right), \\
& b_{k}^{\top} A c_{l}=b_{k}^{\top} A d_{l}=0 \quad(\text { for all } k, l), \\
& c_{l}^{\top} A d_{l^{\prime}}=0 \quad\left(\text { for all } l, l^{\prime}\right),
\end{aligned}
$$

where $k, k^{\prime}$ index the inertial zero normal modes and $l, l^{\prime}$ index the special zero normal modes. For example, Eq. (46d) is equivalent to the condition that $u_{1 l}^{\top} u_{2 l^{\prime}}=0$ for all pairs of special zero normal modes $l, l^{\prime}$. As for the positive normal modes, if we have a block of $r$ degenerate modes at frequency $\omega>0$, we can, without breaking the conditions (10a) and (10b), choose them in such a way that the Hermitian $r \times r$ matrix $[\mathrm{A}]_{i j}=\left(w_{1 i}+i w_{2 i}\right)^{\dagger} A\left(w_{1 j}+i w_{2 j}\right)$ is diagonal. Here $i, j$ index those modes that are part of the degenerate block. Again, we must use $A=\left\langle\Omega^{\top} \Omega\right\rangle$. As a result, the components $w_{1 i}$ and $w_{2 i}$ satisfy

$$
\begin{array}{ll}
w_{1 i}^{\top} A w_{1 j}+w_{2 i}^{\top} A w_{2 j}=0 & (\text { for } i \neq j), \\
w_{1 i}^{\top} A w_{2 j}-w_{2 i}^{\top} A w_{1 j}=0 & (\text { for all } i, j) .
\end{array}
$$

For example, Eq. (47b) is equivalent to the condition that $u_{1 i}^{\top} u_{2 j}-u_{2 i}^{\top} u_{1 j}=0$ for all pairs of positive normal modes $i, j$ that are part of the degenerate block.

\section{DYNAMICAL MAGNETIC SUSCEPTIBILITY}

Response functions, such as dynamical magnetic susceptibilities, allow comparison of calculated spectra to experimental observables (see, for example, Ref. [20]). The dynamic susceptibility $\chi(\tilde{\omega})$ is defined as the complex amplitude of the magnetization that results in linear response from some applied field, divided by the complex amplitude of this applied field. Here $\tilde{\omega}$ is the driving frequency. Dynamical magnetic susceptibility functions can be related directly to the eigenmodes of the dynamical matrix in the presence of damping [29,52]. Using the expressions in Sec. V, our scheme allows one to calculate dynamical susceptibility functions in a way that is usually much less computationally expensive than with spin-dynamics simulations. We do not make any further assumptions on the system (in particular, we do not assume that $M$ and $\Omega$ commute), and we (optionally) take into account the corrections to the mode vectors $u_{1 k}^{\prime}$ and $u_{2 k}^{\prime}$. Our expressions include the contributions from special and inertial zero modes as well as positive modes. 
If we introduce a time-dependent external field $\mathbf{f}_{i}(t)$ into Eq. (32), we get

$$
\dot{\mathbf{m}}_{i}=-\tilde{\gamma} \mathbf{m}_{i} \times\left[-\nabla_{\mathbf{m}_{i}} \mathcal{H}+\mathbf{f}_{i}(t)-\eta \dot{\mathbf{m}}_{i}\right] .
$$

Linearization of this equation near the equilibrium configuration $m_{0}$ gives

$$
\dot{x}=M x+\Omega f(t)-\eta \Omega \dot{x},
$$

with $M, \Omega$, and $x$ defined as in Eq. (33). We can rewrite this as

$$
\dot{x}-M^{\prime} x=g(t),
$$

with $M^{\prime}$ as defined in Eq. (34) and

$$
g(t)=\left(I_{3 n}+\eta \Omega\right)^{-1} \Omega f(t) .
$$

Now let us write

$$
\begin{aligned}
& x(t)=\sum_{k}\left[x_{1 k}(t) u_{1 k}^{\prime}+x_{2 k}(t) u_{2 k}^{\prime}\right], \\
& g(t)=\sum_{k}\left[g_{2 k}(t) u_{1 k}^{\prime}-g_{1 k}(t) u_{2 k}^{\prime}\right] .
\end{aligned}
$$

Notice that we express $x(t)$ and $f(t)$ in terms of the mode vectors $u_{1 k}^{\prime}, u_{2 k}^{\prime}$ that are corrected for damping, which means that $x_{1 k}, x_{2 k}$ are not identical to the canonical variables $p_{k}, q_{k}$. However, for small damping we have

$$
\begin{aligned}
& x_{1 k}(t)=p_{k}(t)+\mathcal{O}(\eta)=-w_{2 k}^{\top} x(t)+\mathcal{O}(\eta), \\
& x_{2 k}(t)=q_{k}(t)+\mathcal{O}(\eta)=w_{1 k}^{\top} x(t)+\mathcal{O}(\eta), \\
& g_{1 k}(t)=u_{1 k}^{\top} f(t)+\mathcal{O}(\eta), \\
& g_{2 k}(t)=u_{2 k}^{\top} f(t)+\mathcal{O}(\eta),
\end{aligned}
$$

where $u_{1 k}, u_{2 k}$ are the unperturbed mode vectors and $p_{k}, q_{k}$ are the corresponding coefficients (12). If the error of $\mathcal{O}(\eta)$ is unacceptable, one could use Eqs. (39) and (42) to calculate the corrected $u_{1 k}^{\prime}, u_{1 k}^{\prime}$ to an error of only $\mathcal{O}\left(\eta^{2}\right)$ and then use Eq. (52).

In terms of the coefficients, Eq. (49) separates into pairs of coupled equations of motion, one pair for each mode. For a positive mode $k$, we have

$$
\begin{aligned}
& \dot{x}_{1 k}+\xi_{k}^{\prime} x_{1 k}+\omega_{k}^{\prime} x_{2 k}=g_{2 k}, \\
& \dot{x}_{2 k}-\omega_{k}^{\prime} x_{1 k}+\xi_{k}^{\prime} x_{2 k}=-g_{1 k} ;
\end{aligned}
$$

for a special zero mode $k$, we have

$$
\dot{x}_{1 k}=g_{2 k}, \quad \dot{x}_{2 k}=-g_{1 k} ;
$$

and for an inertial zero mode $k$, we have

$$
\dot{x}_{1 k}+\xi_{k}^{\prime} x_{1 k}=g_{2 k}, \quad \dot{x}_{2 k}-x_{1 k}=-g_{1 k} .
$$

If we now assume that the system is driven with a driving frequency $\tilde{\omega}$, so that $g_{1 k}(t)=g_{1 k} e^{i \tilde{\omega} t}$ and similarly for $g_{2 k}(t)$ and $x_{1 k}(t), x_{2 k}(t)$ (particular part of the solution), we find

$$
x_{1 k}=\chi_{11 k} g_{1 k}+\chi_{12 k} g_{2 k}, \quad x_{2 k}=\chi_{21 k} g_{1 k}+\chi_{22 k} g_{2 k},
$$

where, if $k$ is a positive mode,

$$
\begin{aligned}
& \chi_{11 k}=\frac{\omega_{k}^{\prime}}{\left(\xi_{k}^{\prime}+i \tilde{\omega}\right)^{2}+\left(\omega_{k}^{\prime}\right)^{2}}, \quad \chi_{12 k}=\frac{\xi_{k}^{\prime}+i \tilde{\omega}}{\left(\xi_{k}^{\prime}+i \tilde{\omega}\right)^{2}+\left(\omega_{k}^{\prime}\right)^{2}}, \\
& \chi_{21 k}=\frac{-\left(\xi_{k}^{\prime}+i \tilde{\omega}\right)}{\left(\xi_{k}^{\prime}+i \tilde{\omega}\right)^{2}+\left(\omega_{k}^{\prime}\right)^{2}}, \quad \chi_{22 k}=\frac{\omega_{k}^{\prime}}{\left(\xi_{k}^{\prime}+i \tilde{\omega}\right)^{2}+\left(\omega_{k}^{\prime}\right)^{2}} ;
\end{aligned}
$$

if $k$ is a special zero mode,

$$
\begin{aligned}
& \chi_{11 k}=0, \quad \chi_{12 k}=-i / \tilde{\omega}, \\
& \chi_{21 k}=i / \tilde{\omega}, \quad \chi_{22 k}=0 ;
\end{aligned}
$$

and if $k$ is an inertial zero mode,

$$
\begin{aligned}
& \chi_{11 k}=0, \quad \chi_{12 k}=\frac{1}{\xi_{k}^{\prime}+i \tilde{\omega}}, \\
& \chi_{21 k}=i / \tilde{\omega}, \quad \chi_{22 k}=-\frac{1}{\tilde{\omega}^{2}-i \xi_{k}^{\prime} \tilde{\omega}} .
\end{aligned}
$$

Suppose that we apply to the system a driving force with a fixed spatial profile $f \in \mathbb{R}^{3 n}$ and driving frequency $\tilde{w}$ and that we are interested in the response of the variable $r=v^{\top} x$, where $v \in \mathbb{R}^{3 n}$ is a fixed vector. For example, if we are interested in the response of total magnetization in the $\hat{\mathbf{x}}$ direction to a uniform external field, also in the $\hat{\mathbf{x}}$ direction, we have $\mathbf{f}_{i}=\hat{\mathbf{x}}$ and $\mathbf{v}_{i}=\hat{\mathbf{x}}$ for all sites $i$. The total dynamical susceptibility is given by

$\chi_{v f}=\sum_{k}\left[c_{v f 11 k} \chi_{11 k}+c_{v f 12 k} \chi_{12 k}+c_{v f 21 k} \chi_{21 k}+c_{v f 22 k} \chi_{22 k}\right]$,

where the amplitudes $c_{v f 11 k}, c_{v f 12 k}, c_{v f 21 k}, c_{v f 22 k}$ can be derived from Eqs. (51) and (52). For small damping, Eqs. (52a) and (53b) give

$$
c_{v f 12 k}=\left(u_{1 k}^{\top} v\right)\left(u_{2 k}^{\top} f\right)+\mathcal{O}(\eta),
$$

and analogously for $c_{v f 11 k}, c_{v f 21 k}$, and $c_{v f 22 k}$.

\section{IMPLEMENTATION}

The procedure for finding the magnetic normal modes can be summarized as follows.

(A) Find a configuration $m=m_{0}$ that is a local minimum of the Hamiltonian $\mathcal{H}$, under the constraint that $\left\|\mathbf{m}_{i}\right\|=$ constant for each position $i$.

(B) If necessary, follow the procedure in Sec. IV to detect and compute any zero normal modes.

(C) Solve the HDGEP of Eq. (29) to find the (low-energy) positive normal modes.

(D) If a damping parameter $\eta>0$ is used, correct the normal modes using the expressions in Sec. V.

All important steps can be efficiently implemented using iterative methods for large Hermitian problems. For concreteness, we shall discuss the iterative methods based on conjugate gradients in a bit more detail. Alternative approaches, such as matrix-free versions of the Lanczos eigenvalue algorithm [43], have similar properties. 
Let us first remark that our scheme can also be used to find the magnetic normal modes near a local energy minimum of some continuum model. One discretizes the system using, for example, the finite-difference method or a geometric finite-element method [53], which gives effective systems that are mathematically equivalent to a finite system [54]. It is important to use a mesh that is smooth enough to avoid effects such as an artificial Peierls pinning of domain walls $[55,56]$. (This effect decreases exponentially in the inverse lattice constant [56], so there is no fundamental problem.)

For completeness, we repeat here the essential properties of the conjugate-gradient methods. In its simplest form, the conjugate-gradient method [57] is an iterative method for solving systems of linear equations, $A x=b$, where $A$ is a symmetric or Hermitian $N \times N$ matrix and $x$ and $b$ are vectors in $\mathbb{R}^{N}$ or $\mathbb{C}^{N} . A$ and $b$ are given; $x$ is asked. The linear system is considered solved when the norm of the residual vector $r=b-A x$ is less than a certain (very small) tolerance. In each iteration $i=1,2, \ldots$, the trial solution $x_{i}$ is updated; its new value $x_{i+1}$ is a certain linear combination of $x_{i}$ and the previous residual vectors $r_{i}, r_{i-1}, r_{i-2}, \ldots$. The method is designed in such a way that only two vectors need to be kept in memory at any given iteration. A more detailed discussion of the algorithm can be found in most textbooks on numerical methods [57]. What is relevant here is the following. (a) We do not need to store the $N^{2}$ elements of $A$. All we need is a routine that can evaluate $A x$ for any given $x$ (the action $x \mapsto A x$ of $A$ ). The conjugate-gradient algorithms use this routine as a "black box." (b) Every trial solution $x_{i}$ is a linear combination of $b, A b, A^{2} b, \ldots, A^{i-1} b$; the conjugate-gradient method is a Krylov-subspace method. A variant of the conjugate-gradient method can be used to solve nonlinear optimization problems [57], where a local minimum of a multivariate function $f(x)$ is asked. Here the gradient $\nabla f$ plays the role of the residual vector. This method is also suitable for minimization problems under constraints $g_{1}(x)=\cdots=g_{k}(x)=0$. In that case, one should project the residual vector $r$ onto the tangent space of the allowable hypersurface in the spirit of the method of Lagrange multipliers.

The conjugate-gradient eigenvalue algorithm [58] can be seen as a special case of constrained nonlinear optimization. If we minimize the function

$$
f(x)=x^{\dagger} D x
$$

under the constraint

$$
g_{1}(x)=x^{\dagger} S x=1 \quad \text { (normalization), }
$$

where $D$ and $S$ are Hermitian matrices, we obtain the lowest eigenvalue $\lambda_{1}$ and the corresponding eigenvector $x_{1}$ of the HDGEP $D x=\lambda S x$. (The SDGEP case, where $D, S$, and $x$ are real, is entirely analogous.) $S$ must be positive definite to guarantee that a minimum exists. Once we have the first eigenvector $x_{1}$, we can obtain the next eigenvector by repeating the minimization under an additional constraint:

$$
g_{2}(x)=x_{1}^{\dagger} S x=0 \quad \text { (orthogonality) }
$$

For $\lambda \neq 0$, this is equivalent to the constraint

$$
g_{2}^{\prime}(x)=\lambda_{1} x_{1}^{\dagger} S x=x_{1}^{\dagger} D x=0 .
$$

Once we have found the second eigenvector, we move on to the third, and so on, applying constraints of the form (66) for all previously obtained eigenvectors. We continue until we have found as many eigenvectors $x_{1}, x_{2}, \ldots$ with eigenvalues $\lambda_{1}<\lambda_{2}<\cdots$ as we need.

The fact that we do not need to explicitly store the matrices in memory is a crucial advantage. For simplicity, let us first consider a one-dimensional $n$-spin chain with only exchange and uniaxial anisotropy energy,

$$
\mathcal{H}=E_{\text {ex }}+E_{\text {ani }}=\sum_{i=1}^{n-1}-2 J \mathbf{m}_{i} \cdot \mathbf{m}_{i+1}-\sum_{i=1}^{n} K m_{i z}^{2} .
$$

The Hessian matrix $A$ (see Sec. III) is given by

$$
A_{(i \alpha)(j \beta)}= \begin{cases}-2 K & \text { if } i=j \quad \text { and } \alpha=\beta=z, \\ -2 J & \text { if } i=j-1, j+1 \text { and } \alpha=\beta, \\ 0 & \text { otherwise; }\end{cases}
$$

equivalently, it may be defined by its action $x \mapsto A x$,

$$
\begin{aligned}
& (A x)_{i \alpha} \\
& \quad= \begin{cases}-2 J\left(x_{(i-1) \alpha}+x_{(i+1) \alpha}\right) & \text { if } \alpha=x, y, \\
-2 J\left(x_{(i-1) \alpha}+x_{(i+1) \alpha}\right)-2 K x_{i \alpha} & \text { if } \alpha=z,\end{cases}
\end{aligned}
$$

where we take $x_{i \alpha}=0$ for $i=0$ and $i=n+1$. We see that the evaluation of the action of $A$ on an arbitrary vector $x$ takes only $\mathcal{O}(N)$ time, while any manipulation with or decomposition of the $3 n \times 3 n$ matrix $A$ obviously takes at least $\mathcal{O}\left(N^{2}\right)$ time if it is explicitly stored in memory in full. That is why Krylov-subspace methods are a popular choice for linear equations or eigenvalue problems of sparse matrices [44]. If long-range interactions are taken into consideration, the matrix $A$ is dense. Nevertheless, the action of $A$ can still be evaluated in much less than $\mathcal{O}\left(N^{2}\right)$ time, as follows. For nearly all physical systems, $A$ can be separated into a short-ranged part $A_{\mathrm{s}}$ such as Eq. (68), which is sparse, and a long-ranged part $A_{1}$, which is invariant under spatial translations (it is a convolution) [29]. To perform the action on a given vector $x$, we separately evaluate the contributions $A_{\mathrm{s}} x$ and $A_{1} x$ and then add them up to obtain $A x=A_{\mathrm{s}} x+A_{1} x$. In typical magnetic systems, the relevant long-range interaction is the dipolar interaction. We can evaluate $A_{1} x$ by performing the convolution in the Fourier representation of $x$, where it becomes a simple elementwise multiplication. The two Fourier transformations that are necessary take $\mathcal{O}(N \log N)$ time [59]. A similar mixed real-space-reciprocal-space approach is taken in most plane-wave electronic-structure codes [60]. Even if the system is not perfectly translationally invariant, for instance, because it has some nonrectangular finite geometry, we can efficiently evaluate $A_{1} x$ by reducing the dipolar problem to the Poisson problem [3] and solving it using multigrid methods [44]. The complexity analysis is similar. It is thus possible to implement a routine that can evaluate $A x$, and hence $M \Omega x(25)$, for any given $x$ in $\mathcal{O}(N \log N)$ rather than $\mathcal{O}\left(N^{2}\right)$ time.

In the remainder of this section, we discuss the specific implementation of each of the four stages listed above.

Stage A. In A minimum-energy configuration $m_{0}$ can be found using, for example, the nonlinear conjugate-gradient 
optimization method, which is implemented in existing micromagnetics codes. Note that many magnetic systems have multiple local energy minima. In this article, we regard one particular $m_{0}$ as given.

Stage B, step (1). In step (1) of Sec. IV, we need to calculate the null vectors $y_{1}, \ldots, y_{s}$ of $\langle M \Omega\rangle$. This is, in fact, a symmetric eigenvalue problem. It might be solved as a particular case of the conjugate-gradient SDGEP algorithm (set $D=\langle M \Omega\rangle$ and $S=I$ ). The sequential nature of this method means that we can efficiently obtain the lowest few eigenvectors. We stop once we find the first positive eigenvalue. The eigenvectors with eigenvalue zero constitute a basis of the null space of $\langle M \Omega\rangle$.

In our definition of the restricted matrix $\langle M \Omega\rangle$, we formally require construction of a basis of the image space of $\Omega$. In practice, we do not normally need to construct the basis explicitly. We may simply set $D=M \Omega$, provided our initial guess $x_{0}$ is in the image space of $\Omega$ (that is, we set $x_{0}=\Omega y_{0}$, where $y_{0}$ is a random vector). Since $x_{0}, M \Omega x_{0}, \Omega x_{0},(M \Omega)^{2} x_{0}$, etc., all lie in the image space of $\Omega$, the minimization will automatically be restricted to trial solutions in this space. We remark that for numerical stability, it may be necessary occasionally to project the trial vector $x_{i}$ back onto the image space of $\Omega$.

Stage B, step (4). In step (4) of Sec. IV, we need to solve the linear system $\langle M \Omega\rangle x=g$, where $g=\langle\Omega\rangle b_{k}$. This problem may seem ill posed, since $\langle M \Omega\rangle$ is not invertible (even with the angular brackets). However, we know that a solution exists ( $g$ lies in the image space of $\langle M \Omega\rangle$ ). Since the solution-vector iterates are always linear combinations of $g,\langle M \Omega\rangle g,(\langle M \Omega\rangle)^{2} g, \ldots$, we in effect restrict our search to trial solutions $x$ in the image space of $\langle M \Omega\rangle$. In this linear subspace, the solution $x$ is unique.

In practice, $g$ will not lie in the image space of $\langle M \Omega\rangle$ numerically exactly, but only up to a small tolerance, so that the solver may fail once the magnitude of the residual vector becomes on the order of this tolerance. We may remedy this as follows. Project $g$ onto the orthogonal complement of $y_{1}, \ldots, y_{s}$, and do the same for $\langle M \Omega\rangle x$ in each iteration. Effectively, we now find a solution of $P\langle M \Omega\rangle P=P h$, where $P$ (symmetric) performs the projection.

For the sake of completeness, we remark that, again, we may use $M \Omega$ instead of $\langle M \Omega\rangle$, as the image space of $M \Omega$ is contained in the image space of $\Omega$.

Stage $C$. The problem (29) can be solved using the conjugate-gradient HDGEP scheme, where in Eq. (26) we set

$$
D=-i\langle\Omega\rangle, \quad S=\langle M \Omega\rangle, \quad \lambda=\omega^{-1} .
$$

Notice that we only need the (action of the) matrices $\Omega$ and $M \Omega$, which have simple forms (24) and (25). Again, we do not need to implement the restrictions $\langle\cdot\rangle$ explicitly, provided that our initial guess is in the image space of $\Omega$. For each positive normal mode (3), there are two solutions of Eq. (26): one with $\lambda=\omega^{-1}$ and one with $\lambda=-\omega^{-1}$. We obviously need to find only one of the two. If we find a negative- $\lambda$ solution $x$, we must take the complex conjugate of $x$ to obtain the positive- $\lambda$ solution. Notice that the eigenvalue $\lambda$ that the HDGEP algorithm finds is the reciprocal of the angular frequency $\omega$. The HDGEP algorithm normalizes the solutions $x$ so that $x_{i}^{\dagger} S x_{j}=x_{i}^{\dagger}\langle M \Omega\rangle x_{j}=\delta_{i j}$. To obtain the correct normalization (30a), we must divide each (positive- $\lambda$ ) solution $x_{i}$ by $\sqrt{\lambda_{i} / 2}$; we have

$$
w_{1 i}+i w_{2 i}=\sqrt{2 / \lambda_{i}} x_{i},
$$

where $w_{1 i}$ and $w_{2 i}$ are the real vectors defined in Eq. (9).

The eigenvalues $\lambda$ at the extremes of the spectrum are $\lambda=$ $-\omega_{0}^{-1}$ and $\lambda=\omega_{0}^{-1}$, where $\omega_{0}$ is the angular frequency of the lowest-frequency positive normal mode. HDGEP algorithms such as the conjugate-gradient scheme find the solutions of Eq. (26) with either the lowest or the highest eigenvalues $\lambda$. We see that it does not matter if we let the algorithm minimize $\lambda$ (as we do above) or maximize $\lambda$ : In either case, we obtain the lowest-frequency normal modes first. If we minimize the eigenvalue $\lambda$, we find the negative- $\lambda$ solutions and we must apply to the trial solution $x$ a constraint $\left(w_{1 k}-i w_{2 k}\right)^{\dagger}(-i\langle\Omega\rangle) x=0$ for each previously obtained positive normal mode $k$ [see Eq. (66)]. If we choose to maximize the eigenvalue $\lambda$, we must apply a constraint $\left(w_{1 k}+i w_{2 k}\right)^{\dagger}(-i\langle\Omega\rangle) x=0$ for each previously obtained positive normal mode $k$. If any zero normal modes were found in stage $\mathrm{B}$, we need to eliminate those from the problem to ensure that $S=\langle M \Omega\rangle$ is positive definite on the space of trial solutions. The constraints (31a) and (31b) that accomplish this are of exactly the same form as the constraints for previously obtained positive normal modes.

The simple conjugate-gradient HDGEP scheme outlined above may be improved in several ways. It is well known that matrix-free eigenvalue methods require good preconditioning to be efficient $[43,44,60,61]$. Indeed, we find that preconditioning as described below greatly improves performance, especially if the exchange constant between adjacent sites is large as compared to the anisotropy constant. This is the case in most atomistic simulations and in continuum systems discretized with a reasonably high spatial resolution. (Only for relatively modest systems, say $n \sim 1000$, is preconditioning unnecessary; methods that use explicit matrix decompositions [42] are likely to be more efficient.) How a preconditioner can be incorporated into the conjugate-gradient HDGEP scheme is described in many texts $[43,44,60,61]$. In addition, efficiency may be improved by using a simultaneous conjugate-gradient scheme $[43,60]$, especially if some of the eigenvalues are closely spaced.

We use a preconditioner that is based on an inversion of the spin-wave dispersion relation (77) in reciprocal space, similar to the preconditioners used to solve the Schrödinger equation in electronic-structure calculations [60]. In other words, the preconditioner approximates the spectrum of the system with the spin-wave spectrum of a homogeneous system and uses this to speed up convergence of the trial solution. Note that since a typical spin-wave dispersion relation has no zeros (see Fig. 4), the preconditioner acts in real space as a convolution with some kernel that decays exponentially, with a characteristic decay distance on the order of the domain-wall width. Thus, we could, in principle, even implement the preconditioner in $\mathcal{O}(N)$ rather than $\mathcal{O}(N \log N)$ time. If the explicit restrictions $\langle\cdot\rangle$ of $M \Omega$ and $\Omega$ are not used, it is, of course, important to ensure that the preconditioned reciprocal vector is projected back onto the image space of $\Omega$ in order to ensure that the trial solution $x$ does not move out of the image space of $\Omega$. 
Preconditioning can also greatly speed up convergence for steps (1) and (4) of stage B.

Stage $D$. In principle, the full set of unperturbed magnetic normal modes needs to be available to calculate the correction due to damping for any given mode. This could be a problem, since we usually know only the normal modes near the bottom of the spectrum. This forces us to truncate the sums in Eqs. (41a), (41b), and (44). We verify in Sec. VIIIF for a realistic system that this approximation is justified. In practice, the high-wave-number spin-wave modes are increasingly oscillatory and have an overlap with the lower, smoother modes that decreases exponentially in wave number.

Notice that the damped modes do not, in general, satisfy the relations (10a) and (10b). To carry out a mode analysis of some configuration near $m_{0}$, first obtain the amplitudes $p_{k}, q_{k}$ of the unperturbed modes using Eq. (11) and then use Eqs. (41a), (41b), and (44) to convert these into the amplitudes of the damped modes.

\section{EXAMPLES}

In this section, we study some key examples that are illustrative of the general properties of magnetic normal modes and make evident the fundamental distinction between inertial and special zero normal modes. We also discuss how to calculate effective masses for the inertial zero normal modes. Figure 2 provides an overview of the spectra of all systems we consider here.

We begin by studying the one-dimensional (1D) spin chain, possibly with a defect, in Sec. VIII A. We specifically look at the effect of damping, and we demonstrate how the expressions in Secs. V and VI can be used to calculate dynamical magnetic susceptibilities. In Sec. VIII B, we discuss how the fundamentally different types of dynamics of magnetic structures can be related to the two types of zero modes (special and inertial). In particular, we show how to calculate effective masses. We focus on the properties of zero modes in spin systems with a 1D or 2D domain wall or a Skyrmion (Secs. VIII C-VIIIE), and we investigate a general relation between zero modes and the dispersion relations of extended systems (Sec. VIIID). Section VIIIF evaluates the accuracy of our perturbative treatment of damping.

The 2D systems are of a size (40000 spins) for which we begin to appreciate the scalability of the iterative HDGEP methods (see Sec. VII). With our code, we are able to find the 20 or 30 lowest modes of these systems in a matter of minutes on just a single CPU core. (We remark that the calculation time could be reduced further by parallelization. Matrix-free iterative methods such as the conjugate-gradient HDGEP scheme, especially the simultaneous versions, are known for being highly parallelizable $[43,44]$.) While for the sake of simplicity the examples only take short-range interactions into account, they could be extended to include magnetostatic (dipolar) and other interactions. This may be done in an efficient manner without any fundamental change to the method (see Sec. VII). Inclusion of magnetostatic interactions in rectangular systems of a similar size would not lead to much longer calculation times, since for the purpose of preconditioning our present code already performs a full FFT of the trial solution in each iteration.

In addition to normal-mode analysis using the method described in Sec. IV, we perform some explicit spin-dynamics simulations for comparison. The simulations referred to in this section are carried out by numerically integrating the LLG equation (32) using a self-written $\mathrm{C}++$ code based on the implicit-midpoint time-stepping scheme [54,62] (without the stochastic term). We always check convergence of our results in the size of the time step $\Delta t$.

\section{A. Spin waves in 1D spin chains}

We first consider a finite, $n$-atom spin chain without defects. We set $\left\|\mathbf{m}_{i}\right\|=m_{\mathrm{S}}$ for all spins. The Hamiltonian $\mathcal{H}=E_{\mathrm{ex}}+$ $E_{\text {ani }}$ consists of nearest-neighbor exchange coupling

$$
E_{\mathrm{ex}}=\sum_{i=1}^{n-1}-2 J \mathbf{m}_{i} \cdot \mathbf{m}_{i+1},
$$

(c) $1 \mathrm{D}$ domain wall (d) $2 \mathrm{D}$ domain wal (e) Skyrmion

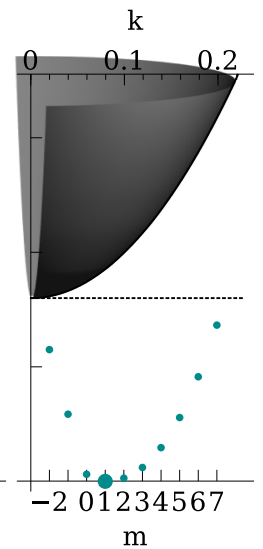

FIG. 2. (Color online) Comparison of the spectra of all systems considered. The presence of defects leads to localized modes with frequencies below the spin-wave continuum. The 1D spin chain is described in Sec. VIII A, the 1D and 2D domain walls in Secs. VIIIC and VIII D, and the Skyrmion in Sec. VIII E. The bottom of the spin-wave continuum is at $\omega=2 \tilde{\gamma} m_{\mathrm{S}} K$ (for uniaxial anisotropy). In the plots the wave vector $k$ is given in units of $a^{-1}$ and the angular frequency $\omega$ in units of $\tilde{\gamma} m_{\mathrm{S}} J$. All continuous branches of modes are discretized (become quasicontinuous) because of the finite dimensions of the systems. 


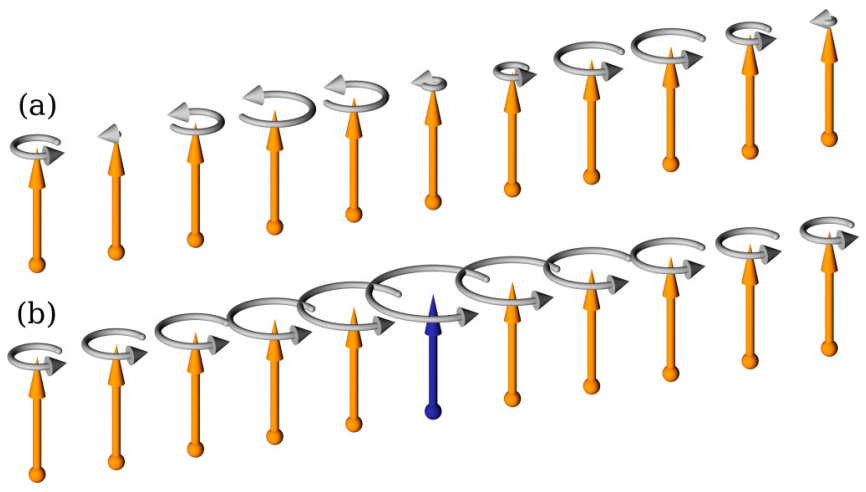

FIG. 3. (Color online) Normal modes of a 1D ferromagnetic spin chain with Neumann boundary conditions. Only a part of the chain is shown. The big straight arrows indicate the equilibrium orientations of the spins. The circular arrows indicate the path traced by the spins if the normal mode is excited. While the normal modes are calculated in the linear (small-amplitude) approximation, we show them with a large amplitude for clarity. (a) A spin-wave mode with $k=0.71 a^{-1}$ in a perfect spin chain. (b) The lowest mode in a spin chain with a defect, located at the site shown in blue, where the anisotropy is reduced from $K=0.45 \mathrm{~J}$ to $K=0.09 \mathrm{~J}$.

with an exchange constant $J>0$ (ferromagnetic) and uniaxial anisotropy

$$
E_{\mathrm{ani}}=\sum_{i=1}^{n}-K\left(\mathbf{m}_{i} \cdot \hat{\mathbf{z}}\right)^{2},
$$

with $K>0$ (easy-axis type). We number the spins as $i=$ $1, \ldots, n$. There is no external magnetic field. We linearize around the uniform, collinear equilibrium configuration $\mathbf{m}_{0 i}=$ $m_{\mathrm{S}} \hat{\mathbf{z}}$, shown in Fig. 3, which is one of the two ground-state configurations ( $\mathbf{m}_{0 i}=-m_{\mathrm{S}} \hat{\mathbf{z}}$ is the other). Our truncation of the exchange couplings (72) at the ends of the chain results in Neumann boundary conditions for the spin waves.

The magnetic normal modes of a 1D spin chain are well known, but we reproduce them here for comparison [see Figs. 2(a), 3(a), 4, and 5(a)]. By taking a general linear

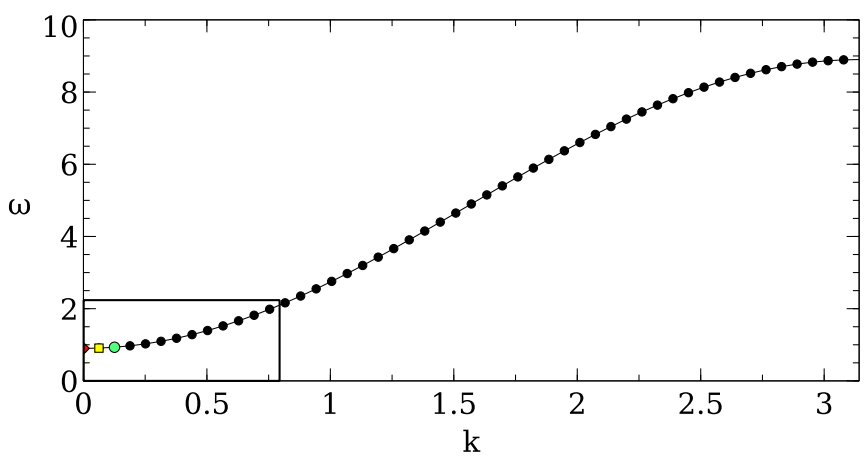

FIG. 4. (Color online) Dispersion $\omega(k)$ of the perfect 1D spin chain. The wave number $k$ is given in units of $a^{-1}$. The angular frequency $\omega$ is given in units of $\tilde{\gamma} m_{\mathrm{S}} J$. The solid line is the analytical dispersion relation (77) and the dots show the spectrum of a 50-spin chain. The Hamiltonian consists of exchange (72) and uniaxial anisotropy (73) with $K=0.45 \mathrm{~J}$. The area in the rectangle is expanded in Fig. 2(a). The colored dots correspond to Fig. 5(a).
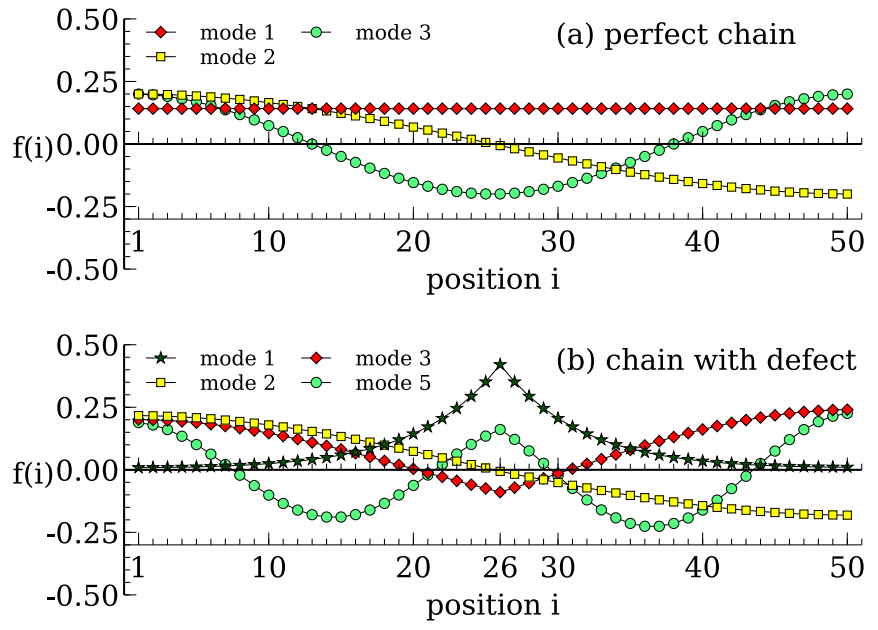

FIG. 5. (Color online) Amplitude profiles $f(i)$ (75) for some low-energy normal modes of a 1D 50-spin chain, (a) without and (b) with a defect. The spin chain with defect is different from the perfect spin chain only at a single site $i=26$, where $K=0.09 \mathrm{~J}$ instead of $0.45 \mathrm{~J}$. Notice that the defect gives rise to a localized mode [see also Figs. 2(b) and 3(b)].

combination of the fundamental solutions (4), we see that the dynamics of any positive normal mode (3) is given by

$$
\mathbf{x}_{i}(t)=A \cos (\omega t+\phi) \mathbf{u}_{1 i}+A \sin (\omega t+\phi) \mathbf{u}_{2 i}+\mathcal{O}\left(A^{2}\right),
$$

where $A$ is the amplitude and $\phi$ is the phase of the mode. The variable $\mathbf{x}_{i}=\mathbf{m}_{i}-\mathbf{m}_{0 i}$ is the deviation of the magnetic moment at site $i$ from its equilibrium position. For the 1D collinear spin chain with Neumann-type boundary conditions, we have spin-wave modes (74) with

$$
\mathbf{u}_{1 i}=f(i) \hat{\mathbf{x}} \quad \text { and } \quad \mathbf{u}_{2 i}=f(i) \hat{\mathbf{y}},
$$

where

$$
f(i)=\cos \left[a k_{l}\left(i-\frac{1}{2}\right)\right]
$$

(standing waves). The dispersion relation is given by

$$
\omega(k)=2 \tilde{\gamma} m_{\mathrm{S}}[K+2 J(1-\cos a k)],
$$

where $k$ is the wave number and $a$ is the spacing between lattice sites. The bottom of the spin-wave continuum is thus at $\omega=2 \tilde{\gamma} m_{\mathrm{S}} K$. The wave number of the mode with index $l=1, \ldots, n$ is given by $k_{l}=\pi(l-1) / a n$. Our code finds the right frequencies $\omega\left(k_{l}\right)$ (see Fig. 4) and the right form of the spin waves [see Fig. 5(a)].

We now consider the effect of a defect, modeled by reducing the anisotropy constant $K$ at a single site. The normal modes are still of the form (75), but have different profiles $f(i)$ [see Fig. 5(b)]. The lowest mode is localized at the defect site and decays exponentially away from it [evanescent spin wave; see also Fig. 3(b)]; its frequency is just below the spin-wave continuum [see Fig. 2(b)]. The other $n-1$ modes are spin-wave modes. They are perturbed with respect to the normal modes of the perfect spin chain. Since in the example of Fig. 5(b) we place the defect almost in the middle $(i=26)$ of a chain of $n=50$ spins, the odd-numbered spin-wave modes have a "kink" at the defect site while the even-numbered 

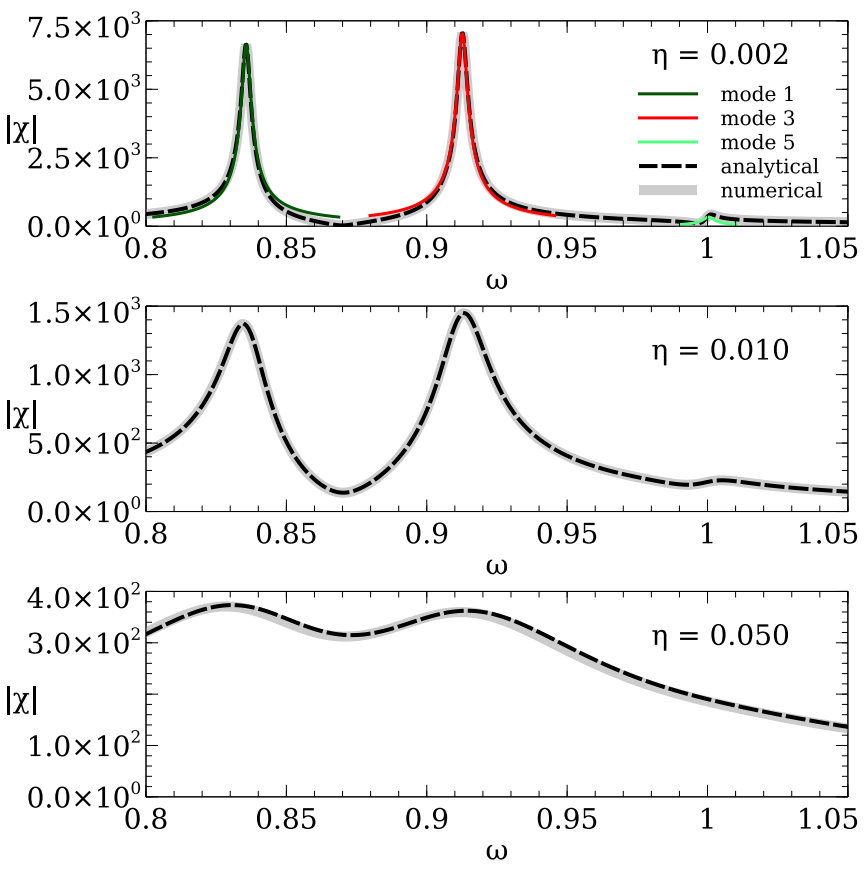

FIG. 6. (Color online) Absolute value of the magnetic susceptibility function $\chi(\omega)$ (driving field perpendicular to equilibrium magnetization) of the 1 D 50-spin chain with a defect $(K=0.09 \mathrm{~J}$ at site $i=26 ; K=0.45 \mathrm{~J}$ everywhere else), for three different values of the damping parameter $\eta$. The driving frequency $\omega$ is given in units of $\tilde{\gamma} m_{\mathrm{S}} J, \chi$ in $J^{-1}$, and $\eta$ in $\left(\tilde{\gamma} m_{\mathrm{S}}\right)^{-1}$. We compare the overall magnetic susceptibility function as obtained in brute-force spin-dynamics simulations to the analytical expression (61), which uses the calculated normal modes. We find excellent agreement. In the first plot, we also show the the absolute value of the contribution of each individual mode. For our particular example, only modes 1, 3, and 5 contribute significantly to the dynamical susceptibility. Other modes generate a magnetization that is negligible when integrated over the whole length of the chain [see, for example, mode 2 in Fig. 5(b)].

spin-wave modes are almost identical to those of the perfect spin chain.

In Fig. 6, we plot the absolute value of the dynamical susceptibility $\chi(\omega)$ of the 50-spin system with defect for several values of the damping parameter $\eta$. Here we consider the response of the magnetization in the $\hat{\mathbf{x}}$ direction (perpendicular to the equilibrium magnetization, which is in the $\hat{\mathbf{z}}$ direction) to an oscillating external magnetic field, also in the $\hat{\mathbf{x}}$ direction. We have obtained $\chi(\omega)$ numerically from spin-dynamics simulations, where we integrate the LLG equation (48) with some small time-dependent driving force. We compare these numerical results to the analytical expression (61) based on the calculated normal modes, taking damping into account to first order. We find an excellent agreement. Higher-order corrections to the eigenfrequencies $\omega_{k}^{\prime}$ under damping probably explain why the actual dynamical susceptibility function for $\eta=0.050 \tilde{\gamma}^{-1} m_{\mathrm{S}}^{-1}$ is very slightly shifted to the left (see also Sec. V). Each peak in the dynamical susceptibility function corresponds to some mode $l$, and its width is proportional to the decay rate $\xi_{l}^{\prime}(35)$, which we can estimate using Eq. (40). We highlight in Fig. 6 the peaks of the most relevant modes of our example system.

\section{B. Inertial versus noninertial behavior of topological defects}

The fundamental distinction between inertial and special zero normal modes described in Sec. II is further clarified by examining the effect of a magnetic field on the dynamics of a topological defect. The general considerations we present here are applied to specific systems in Secs. VIII C-VIII E.

Zero modes typically appear as a consequence of a broken continuous symmetry of the system. For example, the energy of a system with a domain wall (see Secs. VIII C and VIII D) or a Skyrmion (see Sec. VIIIE) in a homogeneous material is invariant under translation of the topological defect. Since no microscopic energy scale is associated with changes of the system that respect the symmetry, weak external perturbations of the Hamiltonian that couple to such changes can have a significant effect over time. By studying the response of the system to such external forces, we establish its effective (that is, low-energy or long-time) dynamics. For example, an effective force on a topological defect may result from an external magnetic field or from dipolar interactions within the system.

Our method establishes a direct link between the normal modes and the most relevant collective coordinates of a topological defect. Even in those systems which are only approximately translationally invariant, such as most systems in which long-range magnetostatic interactions are important, an analysis of the zero and positive normal modes in the absence of magnetostatic interactions is a useful starting point for a collective-coordinate analysis of the dynamical behavior in a weak magnetic field, which can be taken into account as an effective force acting on the defect. We demonstrate this approach in the examples of Secs. VIII C-VIII E.

We consider the dynamics of just a single degree of freedom, corresponding to a zero normal mode $\left(u_{1}, u_{2}\right)$. The deviation $x=m-m_{0}$ of the system from its equilibrium configuration is given by [see Eq. (12)]

$$
x=p_{q} u_{1}+q u_{2}+\mathcal{O}\left(p_{q}^{2}+q^{2}\right) .
$$

We write $p_{q}$ instead of just $p$ to emphasize that this variable is canonically conjugate to $q$. Let us for concreteness assume that the vector $u_{2}$ generates an infinitesimal translation of a topological defect. Thus we have, for a certain constant $\alpha$,

$$
s=\alpha q,
$$

where $s$ is the position of the center of the defect, in units of length. Using Eq. (78), it is straightforward to obtain the coefficient $\alpha$ from the calculated normal mode. The variable canonically conjugate to $s$ is

$$
p_{s}=\alpha^{-1} p_{q}
$$

Let us first consider the case that the zero normal mode is inertial. The unperturbed Hamiltonian is then given, to second order, by [see Eq. (13)]

$$
\mathcal{H}^{\text {iner }}=\frac{1}{2} p_{q}^{2}=\frac{1}{2} \alpha^{2} p_{s}^{2}=p_{s}^{2} /\left(2 m_{\mathrm{eff}}\right),
$$

where we have

$$
m_{\mathrm{eff}}=\alpha^{-2}
$$


the effective mass of the degree of freedom. Suppose that the Hamiltonian (81) is perturbed by an external potential $V(s)$ which depends only on the position of the defect, so that we have $\mathcal{H}=\mathcal{H}^{\text {iner }}+V(s)$. We get

$$
\ddot{s}=\frac{d}{d t} \frac{\partial \mathcal{H}}{\partial p_{s}}=\frac{1}{m_{\mathrm{eff}}} \dot{p}_{s}=-\frac{1}{m_{\mathrm{eff}}} \frac{\partial \mathcal{H}}{\partial s}=-\frac{1}{m_{\mathrm{eff}}} \frac{d V}{d s},
$$

which is Newton's equation of motion.

For a special zero normal mode, the picture is different. The unperturbed Hamiltonian is then given, to second order, by [see Eq. (13)]

$$
\mathcal{H}^{\text {spec }}=0,
$$

which implies, in a sense, an infinite effective mass. Since for the special zero mode no energy term is associated with $p_{s}$, an effective force in the $s$ direction does not, by itself, cause an acceleration in the $s$ direction. It does generate a motion in the canonically conjugate variable; however, here the first, not second, time derivative is proportional to the force. Let us consider a case where $p_{q}$ and $q$ correspond to orthogonal displacements of a 2D magnetic defect, such as a Skyrmion (see Sec. VIII E). We have, for certain constants $\alpha$ and $\beta$,

$$
s_{x}=\alpha q, \quad s_{y}=\beta p_{q},
$$

where $s_{x}$ and $s_{y}$, respectively, represent the $x$ and $y$ coordinate of the position of the defect. Again, we can straightforwardly obtain $\alpha$ and $\beta$ from the calculated normal mode using Eq. (78). If the Hamiltonian (84) is perturbed by an external potential $V\left(s_{x}, s_{y}\right)$, we get

$$
\begin{aligned}
& \dot{s}_{x}=\alpha \beta\left(\partial V / \partial s_{y}\right), \\
& \dot{s}_{y}=-\alpha \beta\left(\partial V / \partial s_{x}\right) .
\end{aligned}
$$

Notice that the velocity (not acceleration) in the $s_{y}$ direction is proportional to the force in the positive $s_{x}$ direction, while the velocity in the $s_{x}$ direction is proportional to the force in the negative $s_{y}$ direction with the same constant of proportionality. We see that we can interpret effective dynamical behavior described by Thiele's equation of motion [14,63] as a direct consequence of the existence of a special zero mode.

\section{1D domain wall}

Even if the Hamiltonian as such is translationally invariant (the material properties are homogeneous), translational symmetry may be broken by the equilibrium configuration $m_{0}$, for instance, if $m_{0}$ contains a domain wall. We consider a 1D spin chain with a domain wall like the one in Fig. 7. We ensure that the equilibrium width of the domain wall is large enough to make the system effectively continuous (Peierls pinning $[55,56]$ is negligible). The $1 \mathrm{D}$ domain wall is the simplest case where the two types of zero modes arise. As in the previous examples, the Hamiltonian consists of exchange and anisotropy terms, which are taken the same for all spins in the system. We consider, however, two types of anisotropy that yield one or the other type of zero mode. We see that the inertial dynamics of many domain walls $[15,64]$ can be interpreted as a consequence of the existence of an inertial zero mode.

For a 1D domain wall, we find below the spin-wave continuum only a single zero mode [see Fig. 2(c)]. If the Hamiltonian is the form considered up to now, with exchange

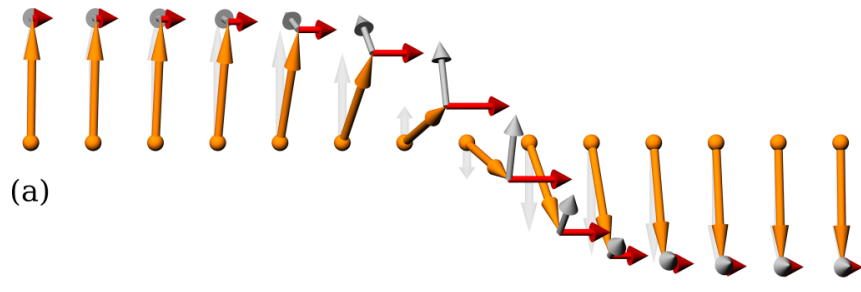

(b)
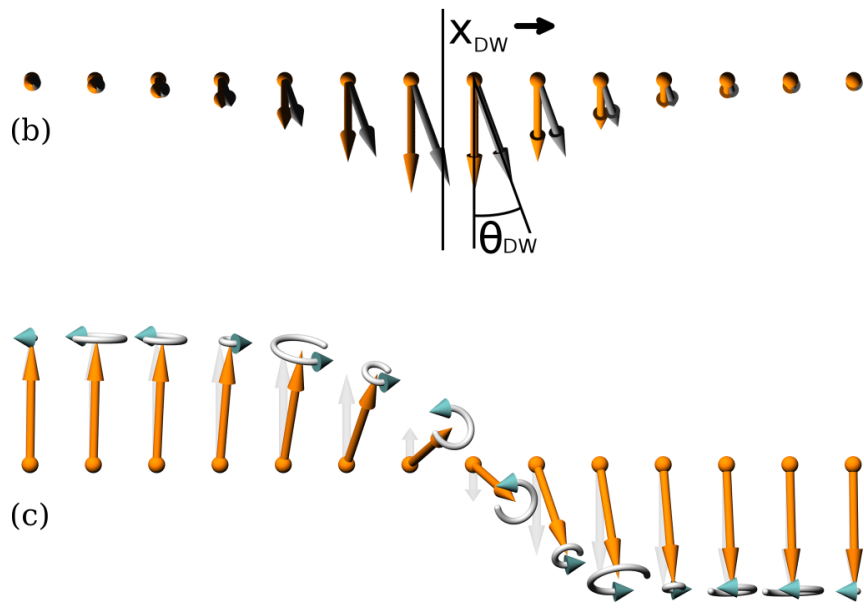

FIG. 7. (Color online) Spin chain with domain wall. The domain wall separates two domains, magnetized in either the positive or the negative $\hat{\mathbf{z}}$ direction. We set $K_{1}=0.04 J$, giving the domain wall a characteristic width [8] of $\delta \propto \sqrt{J / K} a=5.0 a$. The spin chain (200 spins, Neumann boundary conditions) may be considered as effectively continuous and effectively infinite. (Only a part of the chain is shown in the picture. We show one in every three spins of this part of the chain.) The big arrows show the equilibrium configuration $m_{0}$. The vectors $u_{1}$ and $u_{2}$ of the zero mode of the domain wall are indicated in (a) with red $\left(u_{1}\right)$ and gray $\left(u_{2}\right)$ arrows. The actual magnitudes of $u_{1}$ and $u_{2}$ depend on the type of mode (special or inertial) and the effective mass. (b) Top view of the domain wall. The position $x_{\mathrm{DW}}$ of the domain wall and the angle $\theta_{\mathrm{DW}}$ are indicated. (c) Spin-wave mode with $k=0.37 a^{-1}$ for $K_{2}=0.004 \mathrm{~J}$.

and uniaxial anisotropy, this mode is a special zero mode. In Fig. 7(a) we show the two components $u_{1}$ and $u_{2}$ of the zero mode. The component $u_{2}$ generates an infinitesimal increase of the position $x_{\mathrm{DW}}$ of the domain wall, whereas $u_{1}$ generates an infinitesimal increase of the angle $\theta_{\mathrm{DW}}$ [see Fig. 7(b)]. An angle $\theta_{\mathrm{DW}}=0$ or $\theta_{\mathrm{DW}}=\pi$ corresponds to a Bloch domain wall, whereas $\theta_{\mathrm{DW}}= \pm \pi / 2$ corresponds to a Néel domain wall [15]. The coordinate $x_{\mathrm{DW}}$ is canonically conjugate [15] to

$$
p_{\mathrm{DW}}=\frac{2 m_{\mathrm{S}}}{a \tilde{\gamma}} \theta_{\mathrm{DW}} \text {. }
$$

If we apply an external magnetic field in the $\hat{\mathbf{z}}$ direction, which adds to the Hamiltonian a contribution (Zeeman energy) of the form

$$
E_{\text {Zeeman }}=\sum_{i=1}^{n}-\mathbf{h} \cdot \mathbf{m}_{i}=\sum_{i=1}^{n}-h_{z}\left(\mathbf{m}_{i} \cdot \hat{\mathbf{z}}\right),
$$

the domain wall experiences an effective force that acts on the $x_{\text {DW }}$ coordinate. In fact, a displacement of the domain wall by one site (distance $a$ ) leads to one more spin aligned along the 
(a) special $\left(\mathrm{K}_{2}=0\right)$
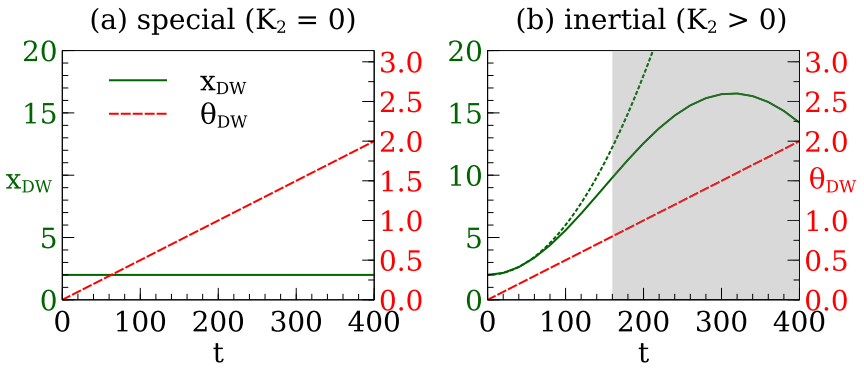

FIG. 8. (Color online) Time evolution of $x_{\mathrm{DW}}$ and $\theta_{\mathrm{DW}}$ in the presence of an external magnetic field $\mathbf{h}=0.005 m_{\mathrm{S}} J \hat{\mathbf{z}}$, for $K_{1}=$ $0.04 \mathrm{~J}$. Due to the external field, the domain wall experiences an effective external potential (89). The position $x_{\mathrm{DW}}$ is given in units of $a$, angle $\theta_{\mathrm{DW}}$ in radians, and time $t$ in units of $\tau=\left(\tilde{\gamma} m_{\mathrm{S}} J\right)^{-1}$. The plots have been obtained in spin-dynamics simulations. (a) Behavior of a domain wall with a special zero mode for $K_{2}=0$. (b) Behavior of a domain wall with an inertial zero mode for $K_{2}=0.016 \mathrm{~J}$. The dotted line is a quadratic fit to the behavior of $x_{\mathrm{DW}}$, which satisfies Newton's law (83) in the limit of small $\theta_{\mathrm{DW}}$. The shaded area indicates where deviations occur (see text).

field and one fewer spin antialigned. This results in an effective external potential

$$
V\left(x_{\mathrm{DW}}\right)=-2 h_{z} m_{\mathrm{S}} x_{\mathrm{DW}} / a .
$$

Nevertheless, the domain-wall position $x_{\mathrm{DW}}$ remains constant, as shown in Fig. 8(a). The conjugated $\theta_{\mathrm{DW}}$ increases linearly (the spins near the center of the domain wall rotate around the $\hat{\mathbf{z}}$ axis). This is in line with the general dynamical behavior predicted for systems with a special zero mode (see Sec. VIII B).

Motion of the domain wall in an external magnetic field along $\hat{\mathbf{z}}$ occurs if we add to the Hamiltonian a term that breaks the symmetry under rotation of the magnetic moments around $\hat{\mathbf{z}}$. In many magnetic systems, magnetostatic interactions favor Bloch domain walls, where the magnetization is in the plane of the domain wall. We model this effect by introducing a second term to the anisotropy energy (73). We use [12,18]

$$
E_{\text {ani }}=\sum_{i}\left[-K_{1}\left(\mathbf{m}_{i} \cdot \hat{\mathbf{z}}\right)^{2}+K_{2}\left(\mathbf{m}_{i} \cdot \hat{\mathbf{x}}\right)^{2}\right],
$$

with $K_{1}, K_{2}>0$. In this case, we find an inertial zero mode, with the components $u_{1}$ and $u_{2}$ again as in Fig. 7(a) but with a different dynamics. Even in the absence of an external field, a small deviation of $\theta_{\mathrm{DW}}$ from its equilibrium value $\theta_{\mathrm{DW}}=0$ now causes a linear motion of the domain wall, $\dot{x}_{\mathrm{DW}}=$ constant. In the presence of an external magnetic field in the $\hat{\mathbf{z}}$ direction, which creates a constant effective force $-\partial V / \partial x_{\mathrm{DW}}=2 h_{z} m_{\mathrm{S}} / a(89)$, we find that $x_{\mathrm{DW}}$ initially increases quadratically in time [see Fig. 8(b)], in perfect agreement with the general dynamical behavior (83) predicted for inertial zero modes.

In Fig. 9 we show how the presence of nonuniaxial anisotropy leads to a finite effective mass, transforming a special zero mode $\left(K_{2}=0\right)$ into an inertial zero mode $\left(K_{2}>\right.$ $0)$. The notion of the effective mass of a domain wall was first introduced by Döring [64]. The deviations from quadratic behavior calculated at large times [shaded area in Fig. 8(b)] are beyond the linearized approach. In principle, the effective

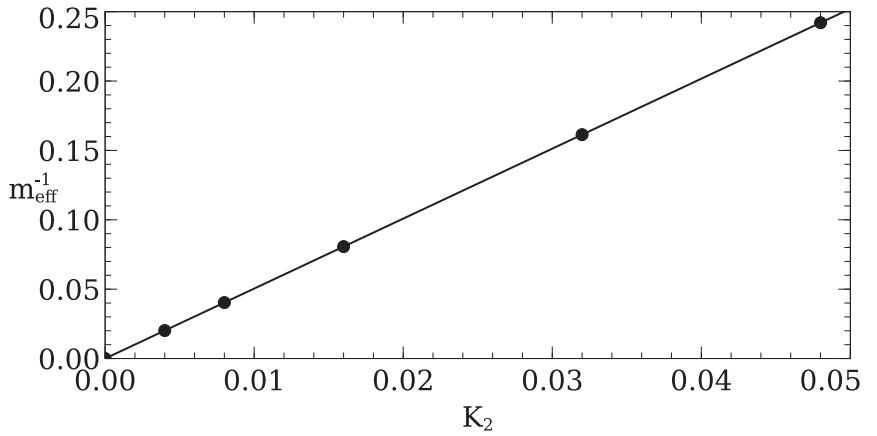

FIG. 9. Inverse Döring effective mass [64] of a domain wall as a function of $K_{2}$, for $K_{1}=0.04 \mathrm{~J}$. We determine the effective masses from the calculated zero modes using Eq. (82). $K_{2}$ is given in units of $J, m_{\text {eff }}^{-1}$ in units of $a^{2} \tilde{\gamma}^{2} J$.

mass, defined as the inverse of the second derivative of the Hamiltonian $\mathcal{H}$ with respect to the momentum $p_{\text {DW }}$ conjugate to $x_{\mathrm{DW}}$, depends on $\theta_{\mathrm{DW}}$. Eventually, in a conservative system the domain wall starts reverting to its original position when $\theta_{\text {DW }}$ reaches $\pi / 2$. This type of motion of the domain wall, which occurs when damping is absent or small as compared to the effective force, is responsible for the phenomenon called Walker breakdown [65].

In addition to the zero mode, we have a spin-wave continuum [see Fig. 2(c)]. In general, it is hard to find analytical solutions of the magnetic normal-mode problem for systems such as these, where the magnetic moments in the equilibrium configuration are not all parallel. However, in this simple case, an analytical solution for the spin-wave modes has been found [13], which we can use to verify the numerical results. In Fig. 10, we compare the calculated spin-wave modes successfully to this analytical solution. It is convenient to express the analytical solution in the coordinate system [13]

$$
\begin{aligned}
m_{j x} & =\left(\cosh \zeta_{j}\right)^{-1} \cos \phi_{j}, \quad m_{j y}=\left(\cosh \zeta_{j}\right)^{-1} \sin \phi_{j}, \\
m_{j z} & =-\tanh \zeta_{j} .
\end{aligned}
$$

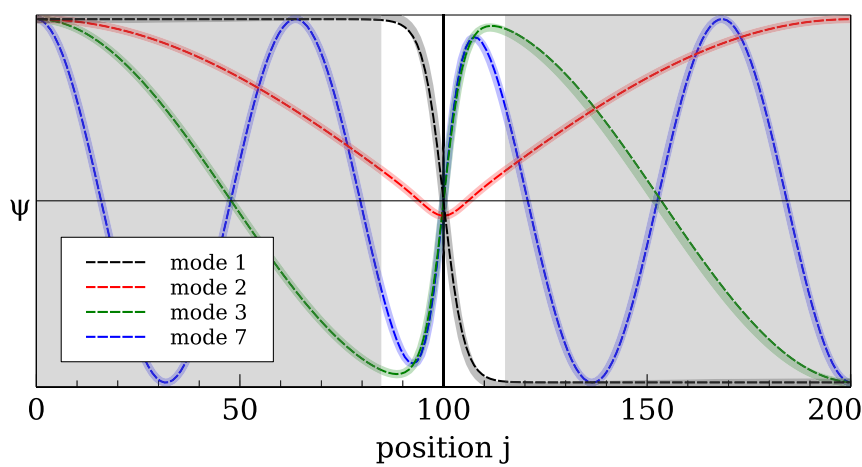

FIG. 10. (Color online) Spin-wave modes in a $1 \mathrm{D}$ uniaxial $\left(K_{1}=\right.$ $\left.0.04 \mathrm{~J}, K_{2}=0\right) 200$-spin chain with a domain wall at the center. We compare the numerical results (thick solid lines) to the analytical form [13] for the continuum model (thin dashed lines). Away from the domain wall, in the shaded area, the spin-wave modes resemble those of a perfect chain and can be characterized by a wave vector $k$. In addition to the spin-wave modes, the system has a localized special zero normal mode [not shown here; see Fig. 7(a)]. 
(a) 2D domain wall
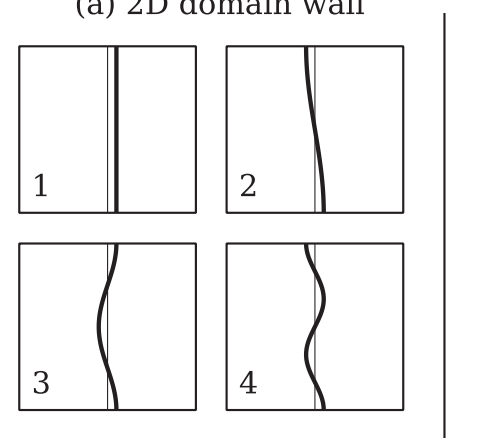

(b) Skyrmion
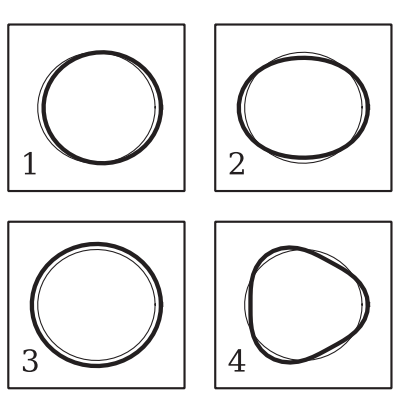

FIG. 11. Sketches of the lowest modes of (a) the 2D domain wall and (b) the Skyrmion. The modes are shown in order of increasing frequency. In both cases, the lowest mode is a zero mode that corresponds to an infinitesimal translation of the defect. For our choice of parameters (see text), the four lowest modes of the Skyrmion are (1) $m=1$, (2) $m=2$, (3) $m=0$, and (4) $m=3$ [see Fig. 2(e)].

In this system the equilibrium configuration $m_{0}$ of the domain wall is given by the linear functions $\zeta_{j}=\left(a j-x_{\mathrm{DM}}\right) / \delta$ and $\phi_{j}=$ constant, where $j$ is the index of the spin, $x_{\mathrm{DM}}$ is the position of the center of the domain wall, and $\delta=\sqrt{J / K} a$ is the characteristic domain-wall length. We convert the Cartesian deviations from the equilibrium orientations, $\mathbf{x}_{j}=$ $\mathbf{m}_{j}-\mathbf{m}_{0 j}$, into values $d \zeta_{j}, d \phi_{j}$ in the coordinate system (91). For any given mode $l$, both functions $d \zeta_{j}, d \phi_{j}$ and both parts $u_{1}, u_{2}$ of the normal mode (3) all have a common shape $f_{j}^{(l)}$, though the amplitudes may be different. We plot $\psi_{j}^{(l)}=f_{j}^{(l)} / \cosh \left(\zeta_{j}\right)$. The fundamental solutions are given by $\psi_{j}=\left[-i k+\tanh \zeta_{j}\right] e^{i k \zeta_{j}}[13]$, where $k \in \mathbb{R}$ is the wave number of the spin wave away from the domain wall, in units of $\delta^{-1}$. In our finite system, the spin-wave spectrum is discretized. We calculate the right $k$ values for the analytical solutions from the numerically obtained values of $\omega$ via Eq. (77). A linear combination of the solutions for $k$ and $-k$ is taken in such a way that a real solution is obtained with a vanishing derivative at the boundaries of the chain.

\section{2D domain wall and Goldstone modes}

If a domain wall is extended to two dimensions, the zero mode of the 1D domain wall turns into a continuum of low-frequency modes $[12,66]$. These modes correspond to bending of the domain wall, as sketched in Fig. 11(a); in other words, they represent small spatial variations of the position $x_{\mathrm{DW}}$ of the domain wall. The domain-wall modes, which form a 1D continuum with a vanishing frequency in the low- $k$ limit, exist alongside the 2D continuum of spin-wave modes [see Fig. 2(d)]. A domain-wall mode can only exist if its frequency is below the bottom of the spin-wave continuum, which puts a maximum on its wave number. The dispersion relation of the domain-wall modes in a system with arbitrary (possibly nonuniaxial) anisotropy was derived in Ref. [12]. Here we show, using very general arguments, that the qualitative features of this dispersion relation follow immediately from the type of zero mode present in the system.

The domain-wall modes are a good example of physically interesting low-energy excitations of large systems, which can

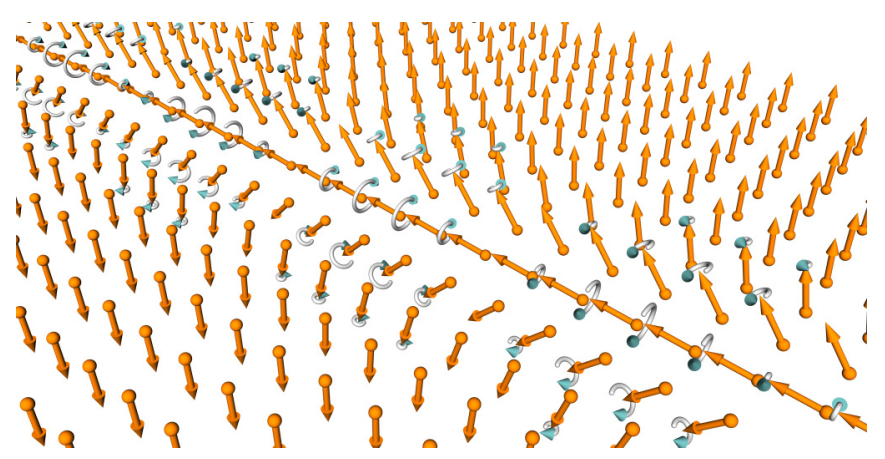

FIG. 12. (Color online) A domain-wall mode. Only one spin is shown for each block of $5 \times 5$ spins; the system $(100 \times 400$ spins $)$ may be considered as effectively continuous. Here we show the 16th domain-wall mode, with wave number $k=15 \pi /(400 a)$. Notice that the motion of the spins is in phase, since the boundary conditions used result in standing waves. When the deviation of the spins at the center of the domain wall from their equilibrium orientations is vertical, the domain wall is bent in a way similar to what is shown in Fig. 11(a). When it is horizontal, the domain wall is not bent; at this point, the energy of the mode is stored as a spatial variation of $\theta_{\mathrm{DW}}$ rather than of $x_{\mathrm{DW}}$.

be found very efficiently using our method. The domain-wall mode in Fig. 12 was calculated in a system of $100 \times 400$ spins (square lattice) with exchange and uniaxial anisotropy $(K=$ $0.04 J$ ). As in Sec. VIII A, our truncation of the expression for the exchange energy results in Neumann boundary conditions. We find that the lowest 26 modes (including the zero mode) of this system are domain-wall modes [see Fig. 2(d)].

The distinction between special and inertial zero modes has important consequences for the dispersion relations of the Goldstone modes that correspond to them, as we show in the following. For the case with uniaxial anisotropy, shown in Fig. 2(d), we see that the zero mode of the domain wall, which is a special zero mode, turns into a continuum with quadratic dispersion. In Fig. 13, we compare this case to a similar system

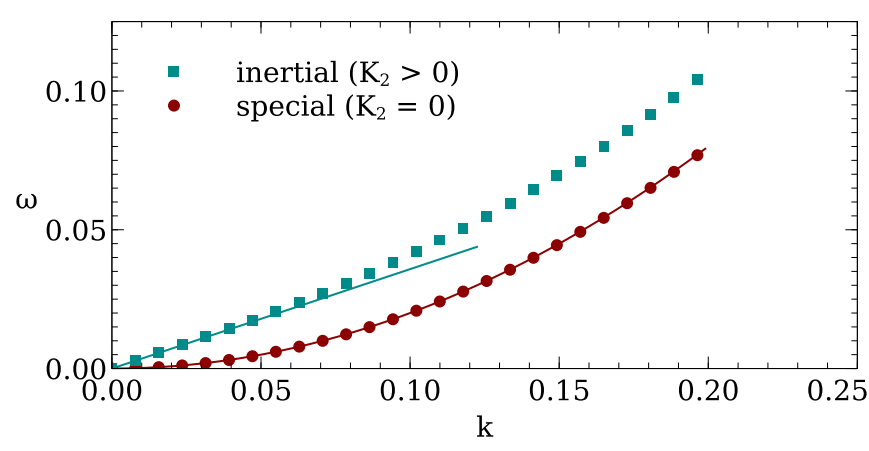

FIG. 13. (Color online) Dispersion curves of the domain-wall modes [12] of a 2D system with uniaxial anisotropy $\left[K_{1}=0.04 \mathrm{~J}\right.$, $K_{2}=0$; see Fig. 2(d)] or nonuniaxial anisotropy $\left(K_{1}=0.04 \mathrm{~J}\right.$, $\left.K_{2}=0.032 J\right)$. The wave number $k$ is in units of $a^{-1}$ and the angular frequency $\omega$ in units of $\tilde{\gamma} m_{\mathrm{S}} J$. In the uniaxial case, where the domain wall has a special zero mode, the dispersion is quadratic. In the nonuniaxial case, where the domain wall has an inertial zero mode, the dispersion is linear in the limit of small $k$. The modes were calculated in a system of $100 \times 400$ spins. 
with nonuniaxial anisotropy. For $K_{2}>0$, where the domain wall has an inertial zero mode, the dispersion relation $\omega(k)$ is linear in $k$ in the limit of low wave number $k$. This suggests that long-wavelength waves in a system with an inertial zero mode propagate with a finite group velocity. Indeed, a finite group velocity is also observed for acoustic waves in crystals, which agrees with the fact that zero modes of systems of coupled point masses are always inertial (see the Appendix).

It is easy to understand the link between the type of zero mode and the low- $k$ behavior of the dispersion relation. Suppose we have a system with a zero mode, such as the 1D spin chain with a domain wall. We describe the relevant dynamics of this system with just two variables, the canonical momentum $p$ and coordinate $q$ (78) corresponding to the zero mode. In the case of the domain wall, $p$ and $q$ are proportional to $x_{\mathrm{DW}}$ and $\theta_{\mathrm{DW}}$, respectively (see Sec. VIII C). We now extend the system to a higher dimension. The variables $p$ and $q$ become functions of position: We have $p(\mathbf{r})$ and $q(\mathbf{r})$. (In the case of the $2 \mathrm{D}$ domain wall, $\mathbf{r} \in \mathbb{R}^{1}$ represents a position along the length of the domain wall.) It is reasonable to assume that for functions $p(\mathbf{r})$ and $q(\mathbf{r})$ that vary very smoothly in $\mathbf{r}$ and for short-range interactions, the Hamiltonian of the system can be approximated by the functional

$$
\mathcal{H}=\int\left[f(p, q)+\frac{1}{2} \rho\|\nabla p\|^{2}+\frac{1}{2} \sigma\|\nabla q\|^{2}\right] d \mathbf{r}
$$

for certain constants $\rho, \sigma>0$. In the limit of small $p$ and $q$ we have that $f(p, q)=0$ for a special zero mode, $f(p, q)=\frac{1}{2} p^{2}$ for an inertial zero mode and $f(p, q)=\frac{1}{2} \omega\left(p^{2}+q^{2}\right)$ for a positive mode [see Eq. (13)]. After Fourier transformation in $\mathbf{r}$, spatial variations with different wave vectors $\mathbf{k}$ decouple and we get

$$
\mathcal{H}_{\mathbf{k}}^{\text {spec }}=\frac{1}{2} \rho k^{2} p_{\mathbf{k}}^{2}+\frac{1}{2} \sigma k^{2} q_{\mathbf{k}}^{2}
$$

for the system with a special zero mode and

$$
\mathcal{H}_{\mathbf{k}}^{\text {iner }}=\frac{1}{2} p_{\mathbf{k}}^{2}+\frac{1}{2} \rho k^{2} p_{\mathbf{k}}^{2}+\frac{1}{2} \sigma k^{2} q_{\mathbf{k}}^{2}
$$

for the system with an inertial zero mode, where we define $k=\|\mathbf{k}\|$. It follows immediately from Hamilton's equations that for $\mathcal{H}_{\mathbf{k}}^{\text {spec }}$ we get

$$
\begin{aligned}
& \dot{p}_{\mathbf{k}}=-\partial \mathcal{H} / \partial q_{\mathbf{k}}=-\sigma k^{2} q_{\mathbf{k}}, \\
& \dot{q}_{\mathbf{k}}=\partial \mathcal{H} / \partial p_{\mathbf{k}}=\rho k^{2} p_{\mathbf{k}},
\end{aligned}
$$

while for $\mathcal{H}_{\mathbf{k}}^{\text {iner }}$ we get

$$
\dot{p}_{\mathbf{k}}=-\sigma k^{2} q_{\mathbf{k}}, \quad \dot{q}_{\mathbf{k}}=p_{\mathbf{k}}+\rho k^{2} p_{\mathbf{k}} .
$$

The momenta $p_{\mathbf{k}}$ can be eliminated from both systems of equations, yielding equations of motion of the form

$$
\ddot{q}_{\mathbf{k}}=-\omega(\mathbf{k})^{2} q_{\mathbf{k}} .
$$

For the special zero mode we get a quadratic dispersion relation

$$
\omega(\mathbf{k})=\sqrt{\rho \sigma}\|\mathbf{k}\|^{2},
$$

whereas for the inertial zero mode we get a linear dispersion relation

$$
\omega(\mathbf{k})=\left[\left(1+\rho\|\mathbf{k}\|^{2}\right) \sigma\right]^{1 / 2}\|\mathbf{k}\|=\sqrt{\sigma}\|\mathbf{k}\|+\mathcal{O}\left(\|\mathbf{k}\|^{2}\right) .
$$

As discussed in Sec. II, a special zero mode naturally arises from a pair of broken continuous symmetries. The above derivation explains why a special zero mode corresponds to a Goldstone mode with a quadratic dispersion relation. The situation is reminiscent of the out-of-plane lattice vibrations (flexural modes) of 2D crystals such as graphene embedded in $3 \mathrm{D}$ space $[67,68]$, which we briefly discuss here. Their quadratic dispersion has been related to a pair of broken continuous symmetries, namely translation and rotation of the graphene sheet in 3D space [69]. (The latter freedom does not exist for 3D crystals embedded in 3D space, whence the linear dispersion relation for traditional acoustic lattice vibrations.) We can model the transversal phonons in 2D crystals using the Hamiltonian

$$
\mathcal{H}=\int\left[\frac{1}{2 m} p_{h}^{2}+\frac{1}{2} \kappa\left(\frac{\partial^{2} h}{\partial x^{2}}\right)^{2}\right] d x,
$$

where $m$ and $\kappa$ are constants, $x$ represents the position along the length of the sheet (for simplicity, we consider variations in one spatial direction only), $h(x)$ displacement in the direction perpendicular to the sheet, and $p_{h}(x)$ linear momentum in the $h$ direction. Paradoxically, a direct application of our method would indicate that this Hamiltonian, like all systems of coupled point masses (see the Appendix), has an inertial zero mode, which would suggest a linear dispersion relation. The reason that this is not the case is, of course, that the energetical penalty on spatial variations is proportional to $\left(\partial^{2} h / \partial x^{2}\right)^{2}$ rather than $(\partial h / \partial x)^{2}$. However, if we write this system in terms of the new variables

$$
\begin{aligned}
p_{q}(x) & =\int-\frac{1}{2} \operatorname{sgn}\left(x-x^{\prime}\right) p_{h}\left(x^{\prime}\right) d x^{\prime}, \\
q(x) & =\partial h /\left.\partial x^{\prime}\right|_{x^{\prime}=x},
\end{aligned}
$$

which can again be shown to be canonically conjugate, the Hamiltonian takes the form

$$
\mathcal{H}=\int\left[\frac{1}{2 m}\left(\frac{\partial p_{q}}{\partial x}\right)^{2}+\frac{1}{2} \kappa\left(\frac{\partial q}{\partial x}\right)^{2}\right] d x .
$$

Since now the Hamiltonian depends only on the spatial derivatives of $p_{q}$ and $q$ and not on $p_{q}$ or $q$ itself, we are back in the situation (93) with a special zero mode.

\section{E. Skyrmion}

Magnetic bubbles and Skyrmions can be seen as circular domain walls (see Fig. 14). The dynamics of a Skyrmion in an effective potential can be understood very well in terms of its normal modes (gyrotropic modes) [14,45,70-72]. We shall see that our algorithm for normal-mode analysis provides a direct way to calculate the effective mass $\mathcal{M}$ and gyrocoupling constant $\mathcal{G}$ of any Skyrmion-like structure, regardless of the details of the interactions present in the system. This is another example of how a normal-mode analysis that includes the zero modes gives the effective equation of motion of some magnetic structure almost immediately.

Skyrmion structures can be stabilized by magnetostatic interactions [9] or by the Dzyaloshinskii-Moriya (DM) interaction [73]. In the latter case, which we consider here, the equilibrium radius is fixed by the material parameters. In the example shown in Fig. 14, we consider a system of $200 \times 200$ spins (square lattice) with only exchange, uniaxial anisotropy 


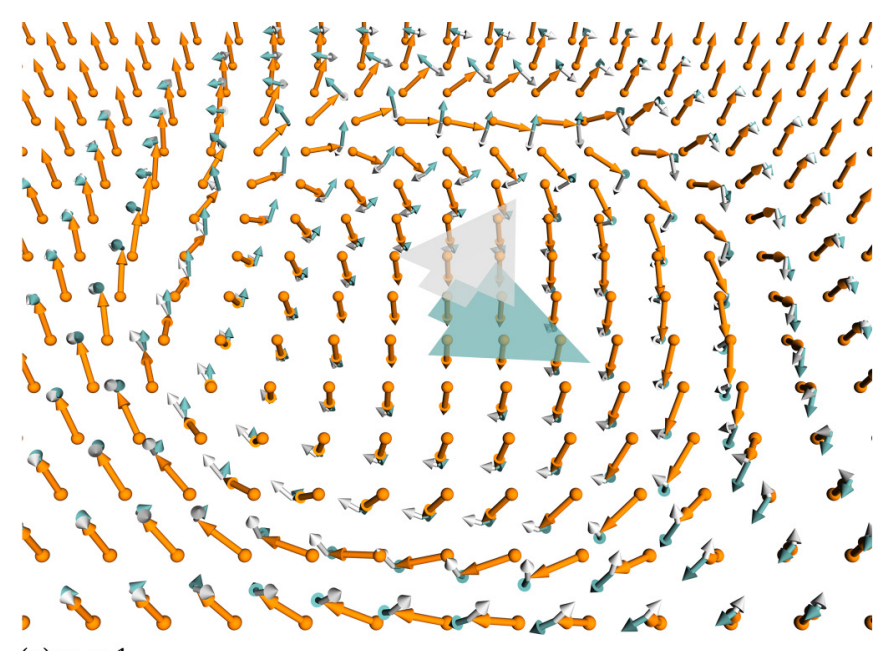

(a) $\mathrm{m}=1$

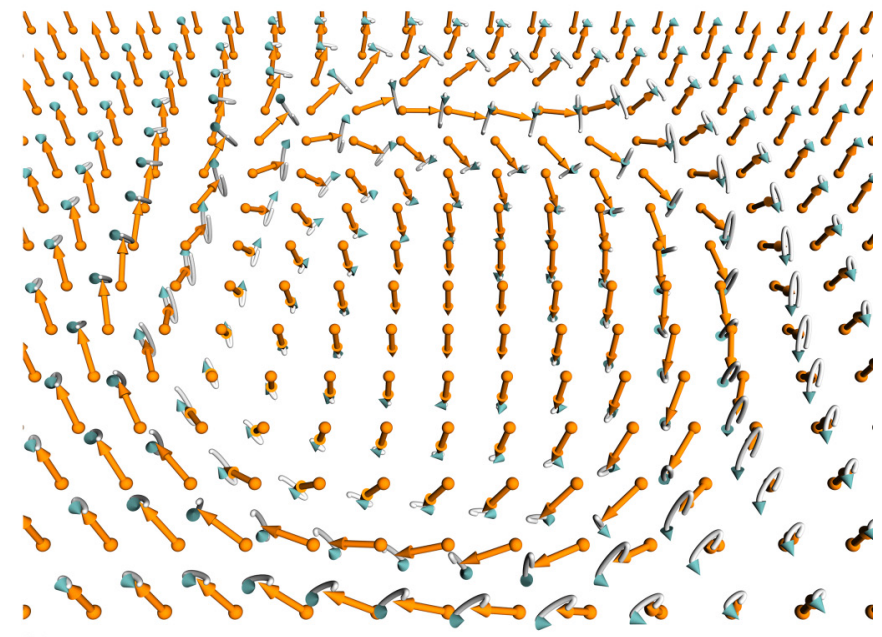

(b) $\mathrm{m}=-1$

FIG. 14. (Color online) A Skyrmion shown with (a) the $m=1$ special zero mode and (b) the $m=-1$ positive mode. Only one spin is shown for each block of $5 \times 5$ spins; the system may be considered as effectively continuous. For our choice of parameters (see text), the $m=-1$ mode is the seventh-lowest mode of the spectrum $(\omega=$ $0.0293 \tilde{\gamma} m_{\mathrm{S}} J$ ).

( $K=0.04 J)$ and DM interactions (no external field). We write the DM interaction as [26]

$$
E_{\mathrm{DM}}=\sum_{\mathbf{x}}-D \mathbf{m}_{\mathbf{x}} \cdot\left(\sum_{\hat{\mathbf{r}}} \hat{\mathbf{r}} \times \mathbf{m}_{\mathbf{x}+a \hat{\mathbf{r}}}\right),
$$

where $\mathbf{m}_{\mathbf{x}}$ is the magnetic moment at the lattice site $\mathbf{x}, \hat{\mathbf{r}}$ sums over the directions of the nearest neighbors in the square lattice $(\hat{\mathbf{r}}=\hat{\mathbf{x}}, \hat{\mathbf{y}},-\hat{\mathbf{x}},-\hat{\mathbf{y}}), a$ is the lattice constant, and $D$ is the interaction strength. Here we set $D=0.125 \mathrm{~J}$. We use periodic boundary conditions. We construct a Skyrmion configuration and relax it. For the given parameters, we get an equilibrium radius of $\sim 27 a$. We find ten normal modes below the spin-wave continuum (edge modes [14]), as shown in Fig. 2(e). We may interpret these modes as domain-wall modes traveling around the Skyrmion [see Fig. 11(b)]. The edge modes can be identified by the number of periods $m$ seen when going around the Skyrmion once. We use a positive or negative value of $m$ to indicate the direction in which the wave travels around the Skyrmion [14]. As pointed out in Ref. [14], the edge-mode spectrum is chiral: It is not symmetric for positive and negative $m$ [see Fig. 2(e)].

The system has translational symmetry in two spatial directions. This symmetry gives rise to a special zero mode $(m=1)$. The vector $u_{1}$ of this mode generates an infinitesimal translation in the $\hat{\mathbf{y}}$ direction and the vector $u_{2}$ generates an infinitesimal translation in the $\hat{\mathbf{x}}$ direction [see Fig. 14(a)]. The existence of this special zero mode suggests that the dynamical behavior of the position of the Skyrmion in an external potential is described by the noninertial equation of motion (86), which is equivalent to Thiele's equation [63] without damping. It has recently been observed that the actual behavior of the Skyrmion position is more accurately described by an equation which has an additional inertial term $[14,74]$, a result which seems to contradict our statement that the Skyrmion possesses a special zero mode and has noninertial character. It is therefore important to make a detailed comparison with the interesting findings of Ref. [14], as we do in the following. An analysis of the normal modes indicates that the inertial term results in this case from the positive mode $m=-1$ [14]. Despite its finite frequency, this mode gives rise to a displacement of the Skyrmion similar to that of the $m=1$ zero mode, albeit with a concomitant change of the Skyrmion configuration [see Fig. 14(b)]. A derivation of the equation of motion of the Skyrmion was given in the Lagrangian formalism in Ref. [14]. Here we reproduce this result in the Hamiltonian formalism and show how the parameters $\mathcal{G}$ and $\mathcal{M}$ of the equation of motion can be obtained immediately from the normal-mode calculation.

The crucial observation, which also underlies the derivation in Ref. [14], is that if we define the position of the Skyrmion as

$$
X=\frac{\int\left(m_{z}(\mathbf{r})-m_{S}\right) x d \mathbf{r}}{\int\left(m_{z}(\mathbf{r})-m_{S}\right) d \mathbf{r}}, \quad Y=\frac{\int\left(m_{z}(\mathbf{r})-m_{S}\right) y d \mathbf{r}}{\int\left(m_{z}(\mathbf{r})-m_{S}\right) d \mathbf{r}},
$$

as was done in Ref. [74], then not only the $m=1$ zero mode [see Fig. 14(a)] but also the $m=-1$ mode [see Fig. 14(b)], which is a positive mode, generates a change in position. In fact, we find from our normal-mode calculation that

$$
\begin{aligned}
& X-X_{0}=\alpha p_{+}+\alpha q_{-}, \\
& Y-Y_{0}=\alpha p_{-}+\alpha q_{+},
\end{aligned}
$$

where $p_{+}, q_{+}$are the canonical momentum and coordinate (12) corresponding to the special zero mode $m=1, p_{-}, q_{-}$correspond to the positive mode $m=-1,\left(X_{0}, Y_{0}\right)$ is the position of the Skyrmion in the equilibrium configuration and $\alpha$ is a constant that can be obtained easily from the calculated normal modes. In our calculation, we find $\alpha=0.282 a \tilde{\gamma}^{1 / 2} m_{\mathrm{S}}^{-1 / 2}$. Since the system is rotationally invariant, the normal modes output by the computer code may be oriented in any direction but we can always rotate them to satisfy Eq. (105). Since we consider only the modes $m= \pm 1$ that couple to position, the unperturbed Hamiltonian is given, to second order, by [see Eq. (13)]

$$
\mathcal{H}=\frac{1}{2} \omega\left(p_{-}^{2}+q_{-}^{2}\right)
$$


where $\omega$ is the angular frequency of the positive mode $m=-1$ (in our example, $\omega=0.0293 \tilde{\gamma} m_{\mathrm{S}} J$ ). There is no energy term associated with the special zero mode $m=1$. If we introduce an external potential that depends only on the position $(X, Y)$, Eq. (106) becomes

$$
\mathcal{H}=\frac{1}{2} \omega\left(p_{-}^{2}+q_{-}^{2}\right)+V(X, Y) .
$$

Using Hamilton's equations, Eq. (107) gives

$$
\begin{aligned}
& \dot{p}_{+}=-\partial \mathcal{H} / \partial q_{+}=-\alpha \partial V / \partial Y, \\
& \dot{q}_{+}=\partial \mathcal{H} / \partial p_{+}=\alpha \partial V / \partial X, \\
& \dot{p}_{-}=-\partial \mathcal{H} / \partial q_{-}=-\omega q_{-}-\alpha \partial V / \partial X, \\
& \dot{q}_{-}=\partial \mathcal{H} / \partial p_{-}=\omega p_{-}+\alpha \partial V / \partial Y,
\end{aligned}
$$

from which it follows that

$$
\begin{aligned}
& \dot{X}=\alpha \dot{p}_{+}+\alpha \dot{q}_{-}=\alpha \omega p_{-}, \\
& \dot{Y}=\alpha \dot{p}_{-}+\alpha \dot{q}_{+}=-\alpha \omega q_{-} .
\end{aligned}
$$

Again taking the time derivative and applying Hamilton's equations, this becomes

$$
\begin{aligned}
& \ddot{X}=\omega \dot{Y}-\alpha^{2} \omega \partial V / \partial X, \\
& \ddot{Y}=-\omega \dot{X}-\alpha^{2} \omega \partial V / \partial Y .
\end{aligned}
$$

These equations of motion are equivalent to Eq. (3) in Ref. [14] if we set

$$
\begin{aligned}
\mathcal{M} & =1 /\left(\alpha^{2} \omega\right), \\
\mathcal{G} & =-1 / \alpha^{2} .
\end{aligned}
$$

The equations of motion (110) consist of a "gyrocoupling" term, which is also present in Thiele's equation, and an additional inertial term, which gives a contribution to the acceleration proportional to the force. For the parameters used in our example, we find $\mathcal{M}=4.29 \times 10^{2} a^{-2} \tilde{\gamma}^{-2} J^{-1}$ and $\mathcal{G}=-12.6 a^{-2} \tilde{\gamma}^{-1} m_{\mathrm{S}} \approx-4 \pi a^{-2} \tilde{\gamma}^{-1} m_{\mathrm{S}}$. For $\mathcal{G}$, an analytical expression was given in Refs. [14] and [63], with which our calculated value is in excellent agreement. From Eqs. (111) and (112) we also recover $\omega=-\mathcal{G} / \mathcal{M}$, which is indeed the frequency of the $m=-1$ mode found in Eq. (4) in Ref. [14] in the absence of an external potential (set $\mathcal{K}=0$ in that equation).

Notice that the above derivation does not contradict the general statement made in Sec. VIII B that a system with a special zero mode should have noninertial dynamics (86). In Eq. (85), we defined the position $\left(s_{x}, s_{y}\right)$ in terms of a perfect translation of the magnetic structure. The positive mode $m=-1$, however, simultaneously induces a change in the configuration of the Skyrmion and is not a perfect translation. In fact, the $m=-1$ mode causes the spins in the center of the circular domain wall to deviate from their Bloch-type equilibrium orientation, which is tangential to the domain wall. This mode therefore does not represent a change in $\left(s_{x}, s_{y}\right)$, while it does represent a change in the Skyrmion position $(X, Y)$ in the sense of Eq. (104). If we define the position according to Eq. (104), one obtains the partially inertial behavior derived above. In many practical situations $(X, Y)$ is the right definition of position, since the effective potential couples to the location of the bubble domain and is mostly insensitive to the domain wall. However, on time scales much longer than $\omega$ the cyclic effect of the positive mode on the position averages out, and $\left(s_{x}, s_{y}\right)$ is again the best representation of the position of the Skyrmion.

\section{F. Accuracy of the corrections to the modes due to damping}

If we introduce damping $(\eta>0)$, this has an effect not only on the amplitudes of the modes, which now decay in time, but also on the mode vectors $u_{1}, u_{2}$ (see Sec. V). Since for large systems we can usually only calculate a number of the lowest modes of the system, which are of the greatest interest, we are forced to truncate the perturbative expressions (41a), (41b), and (44) for these corrections to those modes that are available. In principle, this approximation is uncontrolled. However, we may argue that modes with very different frequencies also have very different characteristic wavelengths and hence have a very small overlap, so that the contribution of high-frequency modes to the damping correction of the low-frequency modes in which we are interested is likely to be negligible. Here we test the accuracy of the damping correction by comparing the actual time evolution of a Skyrmion system to the linearized solutions (36) obtained from normal-mode analysis. This also serves as a test of the expressions (40), (41a), and (41b).

We consider the time evolution of an initial configuration $m=m_{0}+A u_{1 k}$, where mode $k$ is given an initial amplitude $A$. The details of the simulated system are specified below. In Fig. 15, we plot the difference between the results of a numerical time integration of the LLG equation (32) and the linearized solution (4) or (36). Since the error in the numerical solution can be made very small, we may use this difference to evaluate the accuracy of the normal modes. The error stems from two sources. First, the linearization of the LLG equation necessary for normal-mode analysis results in an error of second order in the amplitude $A$. Second, the fact that the
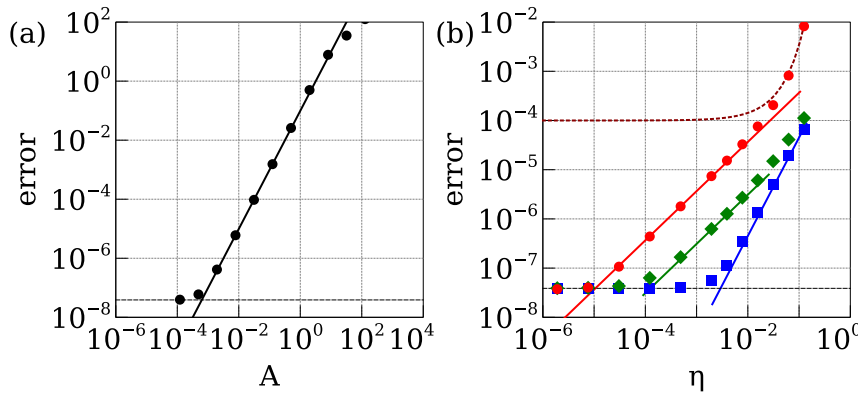

FIG. 15. (Color online) Accuracy of the linearized solution of an initial-value problem for the LLG equation (see text). (a) Error as a function of the amplitude $A$ for zero damping. (b) Error as a function of the damping parameter $\eta$ for a very small, fixed initial amplitude $A=1 \times 10^{-4} m_{\mathrm{S}}^{1 / 2} \tilde{\gamma}^{-1 / 2}$. We consider three different levels of correction for damping in the linearized solution. Red circles, damping is not taken into account at all; green diamonds, the decay rate $\xi^{\prime}$ from first-order perturbation theory is taken into account, but the zero-damping modes $\left(u_{1}, u_{2}\right)$ are used; blue squares, both the modes $\left(u_{1}^{\prime}, u_{2}^{\prime}\right)$ and the decay rate $\xi^{\prime}$ are corrected to first order of perturbation theory. For a fair comparison between different values of $\eta$, we have scaled the errors by the decay factor between the initial and final amplitudes, as indicated by the upper horizontal curve. The lower horizontal line indicates the accuracy of the numerical time-stepping solution. 
modes of a damped system are calculated in perturbation theory gives an additional error that depends on the damping parameter $\eta$.

Figure 15(a) shows the error as a function of the amplitude $A$ for a conservative spin system $(\eta=0)$. We find a quadratic dependence, as expected (1). Figure 15(b) shows the error as a function of $\eta$, for an amplitude $A$ that is chosen so small that the error from nonlinearity is smaller than the error in the numerical solution. The error that we see in the plot is thus due to the error in the perturbative solution of the damped modes. We see that if we do not take damping into account at all (4), the error in the solution is of first order in $\eta$, as expected. If we take damping into account by using the calculated decay rate $\xi^{\prime}(36)$, but without correcting the modes $\left(u_{1}, u_{2}\right)$, the error is much smaller, but it is still of first order in $\eta$. If we also correct the modes $\left(u_{1}^{\prime}, u_{2}^{\prime}\right)$, so that we use the full first-order perturbation theory, we get an error of second order in $\eta$. Notice that we get this order of accuracy even though we use only the contributions of the lowest modes to the perturbative correction. We conclude that, at least in this case, any first-order error due to this truncation is so small as to be insignificant.

The results in Fig. 15 are obtained in a system of $86 \times 86$ spins (square lattice) with uniaxial anisotropy $(K=0.12 J)$ and the DM interaction (103) $(D=0.20 J)$. We use an equilibrium configuration $m_{0}$ containing a single Skyrmion. After relaxation, the Skyrmion is similar to the one in Fig. 14 but has a different radius (owing to the different interaction parameters used). We construct a configuration $m=m_{0}+$ $A u_{1 k}$ in which the second mode $\left(\omega=3.56 \times 10^{-2} \tilde{\gamma} m_{\mathrm{S}} J\right.$, $\left.\xi^{(1)}=1.18 \times 10^{-1} \tilde{\gamma} m_{\mathrm{S}} J\right)$ is given a finite initial amplitude $A$. The magnitudes of the magnetic moments are normalized to $m_{\mathrm{S}}$. We then numerically integrate the LLG equation (32) starting from this initial configuration with $\Delta t=0.1 \tau$ where $\tau=\left(\tilde{\gamma} m_{\mathrm{S}} J\right)^{-1}$. The simulated time is $300 \tau$.

The above results suggest that it is useful to take into account damping in a calculation of the normal modes. Using the expressions in Sec. V, this can be done relatively easily and at a low computational cost. It is already very useful to take into account the decay rate $\xi^{\prime}$ calculated to first order. An even better accuracy can be achieved by also using the first-order corrections to the modes $\left(u_{1}^{\prime}, u_{2}^{\prime}\right)$. We find that in practice, we get an error of second order in $\eta$ in the time evolution of a low-frequency mode even when only a relatively small set of other low-frequency modes were used to calculate the correction. The results also suggest that it is unnecessary to go beyond first-order perturbation theory for damping unless $\eta$ is unusually large.

\section{CONCLUDING REMARKS}

Using explicitly the symplectic structure of the dynamical spin system, we have developed a method that allows us to solve the magnetic normal-mode problem in a very general situation, with the only assumption that the equilibrium magnetic structure corresponds to a local minimum of the Hamiltonian. The examples we have considered (1D and 2D domain walls, Skyrmions) clearly demonstrate that zero modes are an essential part of this normal-mode analysis and can be very useful for understanding dynamics.
Systems with zero modes were difficult to treat within the framework of previous approaches for the magnetic normalmode problem. To our knowledge, all previous approaches assume that the dynamical matrix of the spin system is diagonalizable. This is not the case when inertial zero modes are present, which may occur even for the 1D domain wall. Our approach allows one to calculate, in an efficient and scalable manner, all magnetic normal modes, including the spin-wave modes and those modes that are related, for example, to the motion of localized or extended defects (domain walls, Skyrmions, ...). For the latter case, we give a clear and computationally efficient procedure to calculate the parameters that determine the motion under external forces, such as effective masses. Last but not least, we have developed an efficient perturbation scheme to take into account dissipation effects and calculate dynamical magnetic susceptibilities.

We believe that this approach can be useful in many further problems of spin dynamics, especially those dealing with the motion of Skyrmions and other defects in the magnetic configuration under an external field, their collision (momentum transfer), pinning, dissipation, and so on.

\section{ACKNOWLEDGMENTS}

We thank A. Secchi, J. H. Mentink, K. Y. Guslienko, and O. Eriksson for useful discussions. This work is part of the research program of the Foundation for Fundamental Research on Matter (FOM), which is part of the Netherlands Organisation for Scientific Research (NWO).

\section{APPENDIX: THE NORMAL-MODE PROBLEM OF HAMILTONIAN SYSTEMS}

In this Appendix, we investigate the general properties of linear and nonlinear Hamiltonian systems and their normal modes. An advantage of our general approach is that it explains why it is possible to reduce the magnetic normal-mode problem to the HDGEP: This is a natural consequence of the symplectic structure of the conservative spin system. Moreover, it means that the same method may be reused for other kinds of Hamiltonian systems.

The best-known example of a linear Hamiltonian system is a set of point masses coupled by harmonic springs. For this system the normal-mode problem can be reduced to the SDGEPin an obvious way (see Sec. 2 of the Appendix). However, the general normal-mode problem of linear Hamiltonian systems is much richer [24]. We consider here the normalmode problem of a linear Hamiltonian system with a postive semidefinite Hamiltonian, as results from the linearization of a general Hamiltonian system near an energy minimum.

This Appendix is organized as follows. In Sec. 1 of the Appendix, we reproduce the definition of a linear Hamiltonian system and consider the types of normal modes that it may have. We also explain how the Hamiltonian structure can be used to efficiently perform mode analysis on a given state vector once the normal modes of the system have been calculated. For comparison, we discuss the well-known special case of a system of coupled point masses in Sec. 2 of the Appendix. In Sec. 3 of the Appendix, we generalize the results to a nonlinear Hamiltonian system, possibly defined on a 
Poisson manifold rather than a symplectic manifold. We show that the linearization of a general Hamiltonian system near a local energy minimum results in a linear Hamiltonian system with a positive-semidefinite matrix $\langle M \Omega\rangle$ (defined in Sec. II).

\section{Linear Hamiltonian systems}

Let us first reproduce the definition of a linear Hamiltonian system on the vector space $\mathbb{R}^{2 n}$. Fix some arbitrary basis set $\mathbf{e}_{1}, \ldots, \mathbf{e}_{2 n}$, and let $x^{1}, \ldots, x^{2 n}$ represent the coefficients of a vector $\mathbf{x}$ in this basis. Let the matrix $\Omega$ be antisymmetric $\left(\Omega^{i j}=-\Omega^{j i}\right.$ ) and invertible. (We relax the latter condition in Sec. 3 of the Appendix.) Then the symplectic form

$$
\omega\left(\mathbf{e}_{i}, \mathbf{e}_{j}\right)=\left(\Omega^{-1}\right)_{i j}
$$

defines a symplectic structure on $\mathbb{R}^{2 n}$. Since symplectic forms are bilinear, Eq. (A1) fixes the value of the form for any pair of vectors. The symplectic structure induces a Poisson bracket,

$$
\left\{x^{i}, x^{j}\right\}=-\Omega^{i j},
$$

between the variables $x_{i}, x_{j}$; more generally, for any two functions $f, g$,

$$
\{f, g\}=-\frac{\partial f}{\partial x^{i}} \Omega^{i j} \frac{\partial g}{\partial x^{j}} .
$$

The condition that $\Omega$ be invertible ensures that the symplectic form on $\mathbb{R}^{2 n}$ is nondegenerate. In the special case that the $x^{i}$ with $1 \leqslant i \leqslant n$ represent canonical momenta and the $x^{i}$ with $n+1 \leqslant i \leqslant 2 n$ represent the corresponding canonical coordinates, $\Omega$ takes the familiar form

$$
\Omega=\left[\begin{array}{cc}
0 & I_{n} \\
-I_{n} & 0
\end{array}\right],
$$

where $I_{n}$ is the identity matrix, and in particular, $\Omega$ is orthogonal $\left(\bar{\Omega}=\Omega\right.$, where we define $\bar{\Omega}=\Omega^{-\mathrm{T}}$ ); but we do not make this assumption in this paper.

It is always possible, in principle, to construct a system of canonical momenta and coordinates of a symplectic vector space. Let us write our momenta and coordinates as linear combinations,

$$
\begin{gathered}
p_{(k)}=-w_{2(k) i} x^{i}, \\
q_{(k)}=w_{1(k) i} x^{i},
\end{gathered}
$$

of the variables $x^{i}$ for certain vectors $w_{1(k)}$ and $w_{2(k)}$. By definition, we must have $\left\{q_{(k)}, p_{(l)}\right\}=\delta_{k l}$ and $\left\{p_{(k)}, p_{(l)}\right\}=$ $\left\{q_{(k)}, q_{(l)}\right\}=0$ for all $k, l$. Using Eq. (A2), this can be rewritten as

$$
\begin{aligned}
& w_{1(k) i} \Omega^{i j} w_{2(l) j}=\delta_{k l}, \\
& w_{1(k) i} \Omega^{i j} w_{1(l) j}=w_{2(k) i} \Omega^{i j} w_{2(l) j}=0
\end{aligned}
$$

[see Eqs. (10a) and (10b)]. As a result, we may decompose an arbitrary state vector $x$ as

$$
\begin{aligned}
x^{i} & =\sum_{k=1}^{n}\left[-\left(w_{2(k) h} x^{h}\right) \Omega^{i j} w_{1(k) j}+\left(w_{1(k) h} x^{h}\right) \Omega^{i j} w_{2(k) j}\right] \\
& =\sum_{k=1}^{n}\left(p_{(k)} \Omega^{i j} w_{1(k) j}+q_{(k)} \Omega^{i j} w_{2(k) j}\right)
\end{aligned}
$$

[see Eqs. (11) and (12)]. The vectors $\mathbf{e}_{p(k)}=\Omega^{i j} w_{1(k) j} \mathbf{e}_{i}$ and $\mathbf{e}_{q(k)}=\Omega^{i j} w_{2(k) j} \mathbf{e}_{i}$ form a symplectic basis of the symplectic vector space.

Let us return to the original system of variables $x^{1}, \ldots, x^{2 n}$ (not necessarily canonical) of our symplectic vector space. We define a (time-invariant) Hamiltonian function

$$
\mathcal{H}=\frac{1}{2} x^{i} H_{i j} x^{j},
$$

where $H$ is symmetric. Using the generalized form of Hamilton's equations and the properties of Poisson brackets, we now derive the equation of motion

$$
\dot{x}^{i}=\left\{x^{i}, \mathcal{H}\right\}=-\Omega^{i j} H_{j k} x^{k},
$$

where the dot denotes the time derivative. We may rewrite Eq. (A10) as

$$
\dot{x}^{i}=M^{i}{ }_{j} x^{j},
$$

with

$$
M=-\Omega H .
$$

We see that for a linear Hamiltonian system, $M \Omega=\Omega^{\top} H \Omega$ is symmetric. Conversely, if a given matrix $M$ is such that $M \Omega$ is symmetric (or equivalently, if $\bar{\Omega} M+M^{\top} \bar{\Omega}=0$ ), it is is called a Hamiltonian matrix [75]. The dynamical system (A11) is then a linear Hamiltonian system on the symplectic vector space defined by $\Omega$. In Sec. 3 of the Appendix, we generalize the result that $M \Omega$ is symmetric to Hamiltonian systems that are nonlinear or for which $\Omega$ is not necessarily invertible.

The matrix $M$ describes the dynamical behavior (A11) of the linear Hamiltonian system. This matrix is not necessarily diagonalizable [24]; its Jordan normal form may contain Jordan blocks of high order. Moreover, the eigenvalues of these blocks, which often but not always appear in pairs or quadruples, may be zero, real, imaginary, or complex. Linear Hamiltonian systems may thus display a wide variety of inequivalent types of motion. An exhaustive list of possibilities is given in Ref. [24]. In this paper, we restrict ourselves to systems where $M \Omega$ is positive semidefinite. Even though this condition considerably limits the forms the normal modes may take, we see that three inequivalent types still need to be distinguished.

It can be shown that any linear Hamiltonian system admits a special symplectic basis in which the Hamiltonian takes its normal form [24,76]. In terms of the momenta $p_{(k)}$ and coordinates $q_{(k)}$ that correspond to this special symplectic basis, the Hamiltonian is a direct sum of simple terms, each of which belongs to one of the families listed in Ref. [24]. Note that many of those types of irreducible terms depend on not just one but two or more pairs of canonical momenta and 
coordinates. Here we consider Hamiltonians that are positive semidefinite, for which the possibilities are more limited. Indeed, we have verified that for all but three types, the irreducible term cannot be positive semidefinite by finding a counterexample where the term takes a negative value. The only three exceptions, which are positive semidefinite, are

$$
\begin{aligned}
\mathcal{H}_{k}^{\text {pos }}\left(p_{(k)}, q_{(k)}\right) & =\frac{1}{2} \omega_{k}\left(p_{(k)}^{2}+q_{(k)}^{2}\right), \\
\mathcal{H}_{k}^{\text {spec }}\left(p_{(k)}, q_{(k)}\right) & =0, \\
\mathcal{H}_{k}^{\text {iner }}\left(p_{(k)}, q_{(k)}\right) & =\frac{1}{2} p_{(k)}^{2},
\end{aligned}
$$

where in Eq. (A13a), $\omega_{k}>0$; a term of this type is in fact positive definite. We introduce the names positive, special zero, and inertial zero, respectively, for the three types of terms that may appear in the normal form of a positive-semidefinite Hamiltonian.

By Hamilton's equations, $\dot{p}_{(k)}=-\partial \mathcal{H} / \partial q_{(k)}$ and $\dot{q}_{(k)}=$ $\partial \mathcal{H} / \partial p_{(k)}$, the three types of terms correspond to the following types of motion:

$$
\begin{aligned}
& \text { positive: }\left\{\begin{array}{l}
\dot{p}_{(k)}=-\omega_{k} q_{(k)}, \\
\dot{q}_{(k)}=\omega_{k} p_{(k)},
\end{array}\right. \\
& \text { special zero: }\left\{\begin{array}{l}
\dot{p}_{(k)}=0, \\
\dot{q}_{(k)}=0,
\end{array}\right. \\
& \text { inertial zero: }\left\{\begin{array}{l}
\dot{p}_{(k)}=0, \\
\dot{q}_{(k)}=p_{(k)} .
\end{array}\right.
\end{aligned}
$$

It follows immediately from Eqs. (A8b) and (A11) that Eq. (A14a) corresponds to a positive normal mode (3) with $\omega=\omega_{k}$, that Eq. (A14b) corresponds to a special zero normal mode (5) and that Eq. (A14c) corresponds to a inertial zero normal mode (7), as defined in Sec. II, if we set $\left(u_{1}^{i}, u_{2}^{i}\right)=$ $\left(\Omega^{i j} w_{1(k) j}, \Omega^{i j} w_{2(k) j}\right)$. An important practical consequence of the fact that the normal modes of a Hamiltonian system form a symplectic basis is that we have a direct expression (A8a) for the decomposition of an arbitrary state vector into a linear combination of the normal modes.

While the special zero normal mode (5) can be interpreted as the $\omega \rightarrow 0$ limit of the positive normal mode (3), the inertial zero normal mode (7) is fundamentally different. One might interpret it as the $\omega \rightarrow 0$ limit of

$$
M \tilde{u}_{1}=\tilde{u}_{2}, \quad M \tilde{u}_{2}=-\omega^{2} \tilde{u}_{1},
$$

which for $\omega>0$ is equivalent to Eq. (3) if one sets $\tilde{u}_{1}=$ $u_{1} / \sqrt{\omega}$ and $\tilde{u}_{2}=\sqrt{\omega} u_{2}$.

Notice that even if the original dynamical variables $x^{i}$ represent canonical momenta and coordinates (which is not necessary), the special canonical momenta $p_{(k)}$ and canonical coordinates $q_{(k)}$ of the normal form are still, in principle, linear combinations of all of the $x^{i}$. There is thus no guarantee that $p_{(k)}$ is a linear combination of the original momenta, or that $q_{(k)}$ is a linear combination of the original coordinates, unless the system is of the special form discussed in Sec. 2 of the Appendix.

\section{Harmonically coupled point masses}

The variety in the types of dynamics that linear Hamiltonian systems display (see Sec. 1 of the Appendix and Ref. [24]) may seem surprising. Such variety is not seen in the archetypal example of a linear Hamiltonian system, a collection of point masses coupled by harmonic springs, for which it is obvious how the normal-mode problem can be cast in the form of a SDGEP. We shall see that this type of system is considerably simplified by the special structure of its Hamiltonian, which is not present in all linear Hamiltonian systems. We discuss the system of coupled oscillators here to show how it is special and to explain why the most common method for solving the normal-mode problem cannot be used in the more general case discussed in Sec. II and Sec. 1 of the Appendix.

The Hamiltonian of a system of harmonically coupled point masses is given by $\mathcal{H}=\sum_{i, j} \frac{1}{2} p_{i}\left(S^{-1}\right)_{i j} p_{j}+\sum_{i, j} \frac{1}{2} q_{i} D_{i j} q_{j}$, where $D$ is the force-constant matrix and $S$ is the mass matrix. The matrix $S$ is positive definite; both matrices are symmetric. In the simplest case, we have $S=m I_{n}$, where $m$ is the mass of a single particle. The variables $p_{i}$ and $q_{i}$ represent the momentum and the displacement of particle $i=1, \ldots, n$. (In multidimensional systems, we let $i$ represent the spatial direction as well as the particle index; this does not affect the mathematical structure.) If we write the state of the system as a single vector,

$$
x=\left[\begin{array}{l}
p \\
q
\end{array}\right] \in \mathbb{R}^{2 n},
$$

the matrix $\Omega$ takes its standard form (A4), since the variables $p_{i}$ and $q_{i}$ form a canonical system. The Hamiltonian takes the form (A9) if we set

$$
H=\left[\begin{array}{cc}
S^{-1} & 0 \\
0 & D
\end{array}\right] .
$$

Notice that $H$ is block diagonal: The Hamiltonian does not contain any terms that couple coordinates to momenta. The equation of motion is given by

$$
\left[\begin{array}{l}
\dot{p} \\
\dot{q}
\end{array}\right]=M\left[\begin{array}{l}
p \\
q
\end{array}\right]=\left[\begin{array}{cc}
0 & -D \\
S^{-1} & 0
\end{array}\right]\left[\begin{array}{l}
p \\
q
\end{array}\right],
$$

where we have used Eq. (A12). The structure of Eq. (A18) is such that we can derive equations of motion for the momenta and for the coordinates separately. For the coordinates, we have

$$
\ddot{q}=S^{-1} \dot{p}=-S^{-1} D q .
$$

The fundamental solutions of this equation may be found by calculating the eigenvectors $q^{*}$, which satisfy $S^{-1} D q^{*}=\lambda q^{*}$. This equation is equivalent to the SDGEP

$$
D q^{*}=\lambda S q^{*} .
$$

If we assume that the Hamiltonian is positive semidefinite, so that the the classification of Sec. 1 of the Appendix is applicable, then $D$ must also be positive semidefinite. We have that $\lambda \geqslant 0$, and the vector pair

$$
\left(\tilde{u}_{1}, \tilde{u}_{2}\right)=\left(\left[\begin{array}{c}
S q^{*} \\
0
\end{array}\right],\left[\begin{array}{c}
0 \\
q^{*}
\end{array}\right]\right)
$$

satisfies Eq. (A15) with $\omega=\sqrt{\lambda}$. If $\omega>0$, this is a positive normal mode (3); if $\omega=0$, it is an inertial zero normal mode (7). Notice that special zero normal modes (5) do not occur in a system of coupled point masses. 
We see that the normal-mode problem of a system of coupled point masses can be reduced to the SDGEP, as is well known. The same is true for the normal-mode problems of the wave equation or in elasticity theory, which have a similar mathematical structure (and are in a sense continuum analogs of systems of harmonically coupled masses). However, the same reduction cannot be applied to arbitrary linear Hamiltonian systems. What makes the system of coupled point masses special is that (a) there is a natural system of canonical variables (the momenta and displacements of the individual masses); (b) in this canonical system, the Hamiltonian is the sum of a kinetic-energy term, which depends only on the momenta, and a potential-energy term, which depends only on the coordinates; and (c) the kinetic-energy term is positive definite. As for the spin system, while it is not hard to construct a system of canonical momenta and coordinates (condition (a); see Ref. [47]), in this system the Hamiltonian generally does not separate into a kinetic-energy and a potential-energy part [condition (b)], especially if the equilibrium configuration is not collinear. One might remark that if the Hamiltonian is positive semidefinite a system of momenta and coordinates that satisfies condition (b) must exist: Such a system is a by-product of the solution of the normal-mode problem (see Sec. 1 of the Appendix). The issue, of course, is that we do not know this system when we start. Moreover, the kinetic-energy term is not guaranteed to be positive definite [condition (c)] unless the Hamiltonian is positive definite. Section IV presents a way in which the normal-mode problem of any linear Hamiltonian system can be reduced to the HDGEP, provided that its Hamiltonian is positive semidefinite.

\section{General Hamiltonian systems}

In this section, we generalize the approach of Sec. 1 of the Appendix in two ways. First, we allow the Hamiltonian system to be nonlinear. Second, we no longer require that the matrix $\Omega$ defining the Poisson bracket at $x=0$ is invertible. In the language of symplectic geometry, the latter generalization means that the Hamiltonian system may be defined on a Poisson manifold rather than a symplectic manifold. While any symplectic manifold is also a Poisson manifold, the converse is not true. The spin system in Cartesian coordinates (see Sec. III) is an important example. We show that even under these relaxed conditions, linearization of the equation of motion of a general Hamiltonian system near an equilibrium point $x^{i}=0$ results in a linear Hamiltonian system. In particular, we show that the matrix $M \Omega$ (see Sec. II) is symmetric. Moreover, we show that $\langle M \Omega\rangle$ can be interpreted as the Hessian matrix at the equilibrium point of the restriction of the Hamiltonian function to the symplectic leaf that contains $x=0$. This implies that $\langle M \Omega\rangle$ is indeed guaranteed to be positive semidefinite, as we require, provided that we linearize at a constrained local minimum of the Hamiltonian.

We fix a nonsingular local system of variables $x^{1}, \ldots, x^{m}$ in such a way that $x^{i}=0$ is an equilibrium point. In this system of variables, we expand the Hamiltonian $\mathcal{H}$ to second order in $x$ as

$$
\mathcal{H}(x)=H_{0}-h_{i} x^{i}+\frac{1}{2} x^{i} A_{i j} x^{j}+\mathcal{O}\left(\|x\|^{3}\right),
$$

for a constant scalar $H_{0}=\mathcal{H}(0)$, vector $h_{i}=-\partial \mathcal{H} /\left.\partial x^{i}\right|_{x=0}$, and symmetric matrix $A_{i j}=\partial^{2} \mathcal{H} /\left.\left(\partial x^{i} \partial x^{j}\right)\right|_{x=0}$. We expand the Poisson bracket to first order as

$$
\left\{x^{i}, x^{j}\right\}=-\Omega^{i j}+K^{i j}{ }_{k} x^{k}+\mathcal{O}\left(\|x\|^{2}\right) .
$$

The properties of the Poisson bracket (antisymmetry, Jacobi identity) give the following conditions on the coefficients of this expansion: $\Omega^{i j}$ must be antisymmetric $\left(\Omega^{i j}=-\Omega^{j i}\right)$; $K^{i j}{ }_{k}$ must be antisymmetric in the first two indices $\left(K^{i j}{ }_{k}=\right.$ $\left.-K^{j i}{ }_{k}\right)$; and we must have [77]

$$
K^{i j}{ }_{l} \Omega^{l k}+K^{j k}{ }_{l} \Omega^{l i}+K^{k i}{ }_{l} \Omega^{l j}=0 .
$$

The last condition follows from the Jacobi identity,

$$
\left\{x_{i},\left\{x_{j}, x_{k}\right\}\right\}+\left\{x_{j},\left\{x_{k}, x_{i}\right\}\right\}+\left\{x_{k},\left\{x_{i}, x_{j}\right\}\right\}=0,
$$

which holds for any Poisson bracket $\{\cdot, \cdot\}$. From Eq. (A23), we get

$$
\begin{aligned}
\left\{x_{i},\left\{x_{j}, x_{k}\right\}\right\} & =-\Omega^{j k}\left\{x^{i}, 1\right\}+K^{j k}{ }_{l}\left\{x^{i}, x^{l}\right\}+\left\{x^{i}, \mathcal{O}\left(\|x\|^{2}\right)\right\} \\
& =K^{j k}{ }_{l} \Omega^{l i}+\mathcal{O}(\|x\|) .
\end{aligned}
$$

Since this expression holds at any point $x$, we obtain Eq. (A24) by collecting the constant parts of the three cyclic permutations of it that appear in Eq. (A25).

Using Eqs. (A22) and (A23) and the general properties of Poisson brackets, we derive the equation of motion to first order from the generalized Hamilton equations,

$$
\dot{x}^{i}=\left\{x^{i}, \mathcal{H}\right\}=\Omega^{i j} h_{j}+M^{i}{ }_{j} x^{j}+\mathcal{O}\left(\|x\|^{2}\right),
$$

where

$$
M_{j}^{i}=-\Omega^{i k} A_{k j}-K^{i k}{ }_{j} h_{k} .
$$

Equation (A28) may be considered as the equivalent of Eq. (21) for a general Hamiltonian system. Since $\dot{x}^{i}=0$ at $x^{i}=0$, we must have $\Omega^{i j} h_{j}=0$. From this fact and Eq. (A24), we can derive that $M \Omega$ is symmetric, as follows. We may write $(M \Omega)^{i j}=F^{i j}+G^{i j}$, where $F^{i j}$ is given by

$$
F^{i j}=-\Omega^{i k} A_{k l} \Omega^{l j}=\Omega^{k i} A_{k l} \Omega^{l j},
$$

and $G^{i j}$ is given by

$$
G^{i j}=-K^{i k}{ }_{l} h_{k} \Omega^{l j}=K^{k i}{ }_{l} h_{k} \Omega^{l j} .
$$

$F^{i j}$ is obviously symmetric ( $A_{i j}$ is symmetric). We can see that $G^{i j}$ is symmetric by rewriting it as

$$
\begin{aligned}
G^{i j} & =\frac{1}{2}\left(K^{k i}{ }_{l} \Omega^{l j}-K^{j k}{ }_{l} \Omega^{l i}-K^{i j}{ }_{l} \Omega^{l k}\right) h_{k} \\
& =\frac{1}{2} K^{k i}{ }_{l} \Omega^{l j} h_{k}+\frac{1}{2} K^{k j}{ }_{l} \Omega^{l i} h_{k}-\frac{1}{2} K^{i j}{ }_{l} \Omega^{l k} h_{k},
\end{aligned}
$$

where we have used Eq. (A24). If $x=0$ is an equilibrium position, Eq. (A27) implies $\Omega^{i j} h_{j}=0$ and the last term vanishes. The other two terms together are explicitly symmetric under $i \leftrightarrow j$.

Except for the fact that $\Omega$ is not necessarily invertible, we could conclude from the symmetry of $M \Omega$ that the linearization $\dot{x}^{i}=M^{i}{ }_{j} x^{j}$ of a general Hamiltonian system near an equilibrium point is a linear Hamiltonian system in the sense of Sec. 1 of the Appendix. To be explicit, the matrix $\Omega$ of this linear Hamiltonian system is defined, according to Eq. (A23), by

$$
\Omega^{i j}=-\left.\left\{x^{i}, x^{j}\right\}\right|_{x=0}=\left.\left\{x^{j}, x^{i}\right\}\right|_{x=0},
$$


which is the value of the Poisson bracket between $x^{j}$ and $x^{i}$ at $x=0$, while the symmetric matrix $M \Omega$ is given by

$$
(M \Omega)^{i j}=-\Omega^{i k} A_{k l} \Omega^{l j}-K^{i k}{ }_{l} h_{k} \Omega^{l j} .
$$

Since $\Omega$ is antisymmetric, its rank is always even. We write $\operatorname{rank}(\Omega)=2 n$. If $m>2 n(\Omega$ is not invertible), we can make $\Omega$ invertible by interpreting the matrices $\Omega$ and $M \Omega$ as restricted to the $2 n$-dimensional image space of $\Omega$. In the notation of Sec. II, we get $\langle\Omega\rangle$ and $\langle M \Omega\rangle$. We may do this because the image space of $M \Omega$ is contained in the image space of $\Omega$. Thus, the matrices $\langle M \Omega\rangle$ and $\langle\Omega\rangle$ together define a proper linear Hamiltonian system.

Our method for the normal-mode problem requires that $\langle M \Omega\rangle$ be positive semidefinite (see Sec. IV). We can show that it is if $x=0$ is a (constrained) local minimum of the Hamiltonian $\mathcal{H}$. For simplicity, we first consider the case $m=2 n$ ( $\Omega$ is invertible). If $\Omega$ is invertible, we have $h=0$, so that $M \Omega=-\Omega A \Omega=\Omega^{\top} A \Omega$. Evidently, $M \Omega$ is positive (semi)definite if and only if $A$, the Hessian matrix of $\mathcal{H}$, is positive (semi)definite. Consequently, if $x=0$ is a local minimum of $\mathcal{H}$, then $M \Omega$ is positive semidefinite.

For $m>2 n$, the dynamical matrix (A28) is no longer determined only by the Hessian matrix $A$ of $\mathcal{H}$; there is an additional $h$-dependent term, which is essential. We shall see that the matrix $\langle M \Omega\rangle$ can be interpreted as the Hessian matrix of the restriction of the Hamiltonian function $\mathcal{H}$ to a certain $2 n$-dimensional submanifold containing $x=0$. For example, while the Hamiltonian $\mathcal{H}=-\mathbf{m} \cdot \hat{\mathbf{z}}$ has no local minimum on $\mathbb{R}^{3}$, is has a constrained minimum at $\mathbf{m}=\hat{\mathbf{z}}$ on the set $S_{c=1}^{2}=\left\{\mathbf{m} \in \mathbb{R}^{3}:\|\mathbf{m}\|=1\right\}$. For positive semidefiniteness of $\langle M \Omega\rangle$ we do not require that $x=0$ be an actual local minimum of $\mathcal{H}$; it is sufficient that $x=0$ be a constrained local minimum on this submanifold. To define the relevant submanifold in a general setting, it is necessary to use some elements from the theory of symplectic structures and Poisson structures [46].

In a symplectic manifold, any point (that is, any state of the system) may be reached from any other point by following the trajectory generated by a suitably chosen Hamiltonian function $\mathcal{H}$, or a finite sequence of such trajectories. In a Poisson manifold, this is not necessarily the case. However, by the splitting theorem on Poisson manifolds [46], a Poisson manifold can be divided into equivalence classes of points for which this is possible. These equivalence classes are symplectic submanifolds of the Poisson manifold and are called symplectic leaves. Two points of a Poisson manifold are in the same symplectic leaf if one can get from one point to the other through a finite sequence of trajectories induced by Hamiltonian functions. For example, consider a conservative spin system (see Sec. III) with a single spin $\mathbf{m} \in \mathbb{R}^{3}$, which is governed by the equation of motion $\dot{\mathbf{m}}=\mathbf{m} \times \nabla \mathcal{H}$. Since this equation conserves $\|\mathbf{m}\|$, a spin in position $\mathbf{m}=\hat{\mathbf{z}}$ will never end up in position $\mathbf{m}=\frac{1}{2} \hat{\mathbf{z}}$, regardless of the choice of $\mathcal{H}$. However, it may at some point in time reach $\mathbf{m}=\hat{\mathbf{y}}$, for instance, if the Hamiltonian is given by $\mathcal{H}=\mathbf{m} \cdot \hat{\mathbf{x}}$. Thus, the Poisson manifold of the conservative single-spin system (that is, $\mathbb{R}^{3}$ equipped with the spin Poisson bracket; see Sec. III) splits into symplectic leaves of the form $S_{c}^{2}=\left\{\mathbf{m} \in \mathbb{R}^{3}\right.$ : $\|\mathbf{m}\|=c\}$ for $c \geqslant 0$. leaf containing the equilibrium point $x=0$ can locally be parametrized by a vector $v_{i}$, which we require to lie in the image space of $\Omega^{i j}$, as

$$
x^{i}=-\Omega^{i j} v_{j}-\frac{1}{2} K^{i j}{ }_{k} \Omega^{k l} v_{j} v_{l}+\mathcal{O}\left(\|v\|^{3}\right)
$$

if we assume that the Poisson bracket of the Poisson manifold is of the form (A23). By substitution of this expression into (A22), we find that in terms of $v$, the Hamiltonian becomes

$$
\begin{aligned}
\mathcal{H}(v)= & H_{0}+h_{i} \Omega^{i j} v_{j}+\frac{1}{2}\left(-\Omega^{i k} A_{k l} \Omega^{l j}-K^{i k}{ }_{l} h_{k} \Omega^{l j}\right) v_{i} v_{j} \\
& +\mathcal{O}\left(\|v\|^{3}\right) .
\end{aligned}
$$

Here we have used that $v=\mathcal{O}(\|x\|)$ : The fact that $v$ lies in the image space of $\Omega$ guarantees $\Omega^{i j} v_{j} \neq 0$ in Eq. (A34). If $x=0$ is an equilibrium point, the linear term in Eq. (A35) vanishes $\left(\Omega^{i j} h_{j}=0\right)$. The matrix of the quadratic term in Eq. (A35), which is identical to the Hessian matrix of the Hamiltonian $\mathcal{H}$ restricted to the symplectic leaf, is identical to $\langle M \Omega\rangle$ (A33). (We must write the angular brackets $\langle\cdot\rangle$ here because $v$ was assumed to lie in the image space of $\Omega$.) Thus, if $x=0$ is a local minimum of $\mathcal{H}$ on the symplectic leaf that contains the point $x=0$, the matrix $\langle M \Omega\rangle$ is positive semidefinite and the method presented in Sec. IV can be used.
[1] W. F. Brown, Micromagnetics (Interscience, New York, 1963).

[2] A. Aharoni, Introduction to the Theory of Ferromagnetism, 2nd ed. (Oxford University Press, USA, 2001).

[3] For a review see, for example J. Fidler, R. W. Chantrell, T. Schrefl, and M. Wongsam, in Encyclopedia of Materials: Science and Technology, edited by K. H. J. Buschow, R. W. Cahn, M. C. Flemings, and B. Ilschner (Pergamon, Oxford, UK, 2001), pp. 5642-5651.

[4] K. Chen and D. P. Landau, Phys. Rev. B 49, 3266 (1994).

[5] B. Skubic, J. Hellsvik, L. Nordström, and O. Eriksson, J. Phys.: Condens. Matter 20, 315203 (2008).

[6] L. D. Landau and E. M. Lifshitz, Phys. Z. Sowjetunion 8, 153 (1935).

[7] T. L. Gilbert, IEEE Trans. Magn. 40, 3443 (2004).

[8] R. Skomski, Simple Models of Magnetism (Oxford University Press, USA, 2008), Chap. 4, pp. 107-148.

[9] T. H. O’Dell, Rep. Prog. Phys. 49, 589 (1986).
[10] A. I. Akhiezer, V. G. Baryakhtar, and S. V. Peletminskii, Spin Waves (North-Holland, Amsterdam, 1968).

[11] S. V. Vonsovsky, Magnetism (Wiley, New York, 1974), Vol. 2.

[12] A. A. Thiele, Phys. Rev. B 7, 391 (1973).

[13] J. S. Helman, H. B. Braun, J. S. Broz, and W. Baltensperger, Phys. Rev. B 43, 5908 (1991).

[14] I. Makhfudz, B. Krüger, and O. Tchernyshyov, Phys. Rev. Lett. 109, 217201 (2012).

[15] G. Tatara, H. Kohno, and J. Shibata, Phys. Rep. 468, 213 (2008).

[16] K. Everschor, M. Garst, B. Binz, F. Jonietz, S. Mühlbauer, C. Pfleiderer, and A. Rosch, Phys. Rev. B 86, 054432 (2012).

[17] S. S. P. Parkin, M. Hayashi, and L. Thomas, Science 320, 190 (2008).

[18] R. Wieser, E. Y. Vedmedenko, and R. Wiesendanger, Phys. Rev. B 81, 024405 (2010). 
[19] X. S. Wang, P. Yan, Y. H. Shen, G. E. W. Bauer, and X. R. Wang, Phys. Rev. Lett. 109, 167209 (2012).

[20] F. Giesen, J. Podbielski, and D. Grundler, Phys. Rev. B 76, 014431 (2007).

[21] F. Montoncello, L. Giovannini, and F. Nizzoli, J. Appl. Phys. 103, 083910 (2008).

[22] G. Gubbiotti, M. Madami, S. Tacchi, G. Carlotti, H. Tanigawa, T. Ono, L. Giovannini, F. Montoncello, and F. Nizzoli, Phys. Rev. Lett. 97, 247203 (2006).

[23] Y. Talbi, P. Djemia, Y. Roussigné, J. BenYoussef, N. Vukadinovic, and M. Labrune, J. Phys.: Conf. Ser. 200, 072107 (2010).

[24] V. I. Arnold, Mathematical Methods of Classical Mechanics, 2nd ed. (Springer, New York, 1989), pp. 381-384.

[25] M.-A. B. W. Bolte, G. D. Meier, M. Najafi, and D. P. F. Möller, in Proceedings of the 20th European Conference on Modelling and Simulation, May 28-31, 2006, Bonn, Germany, edited by W. Borutzky, A. Orsoni, and R. Zobel (European Council for Modelling and Simulation, Bonn, Germany, 2006), p. 487.

[26] M. Mochizuki, Phys. Rev. Lett. 108, 017601 (2012).

[27] R. Arias and D. L. Mills, Phys. Rev. B 63, 134439 (2001).

[28] M. Grimsditch, L. Giovannini, F. Montoncello, F. Nizzoli, G. K. Leaf, and H. G. Kaper, Phys. Rev. B 70, 054409 (2004).

[29] S. Labbé and P.-Y. Bertin, J. Magn. Magn. Mater. 206, 93 (1999).

[30] F. Boust and N. Vukadinovic, Phys. Rev. B 70, 172408 (2004).

[31] R. Zivieri and G. Consolo, Adv. Condens. Matter Phys. 2012, 765709 (2012).

[32] S. Tacchi, F. Montoncello, M. Madami, G. Gubbiotti, G. Carlotti, L. Giovannini, R. Zivieri, F. Nizzoli, S. Jain, A. O. Adeyeye, and N. Singh, Phys. Rev. Lett. 107, 127204 (2011).

[33] S. Tacchi, M. Madami, G. Gubbiotti, G. Carlotti, H. Tanigawa, T. Ono, and M. P. Kostylev, Phys. Rev. B 82, 024401 (2010).

[34] L. Giovannini, F. Montoncello, F. Nizzoli, G. Gubbiotti, G. Carlotti, T. Okuno, T. Shinjo, and M. Grimsditch, Phys. Rev. B 70, 172404 (2004)

[35] F. Montoncello, L. Giovannini, F. Nizzoli, H. Tanigawa, T. Ono, G. Gubbiotti, M. Madami, S. Tacchi, and G. Carlotti, Phys. Rev. B 78, 104421 (2008).

[36] R. Zivieri, F. Montoncello, L. Giovannini, F. Nizzoli, S. Tacchi, M. Madami, G. Gubbiotti, G. Carlotti, and A. O. Adeyeye, Phys. Rev. B 83, 054431 (2011).

[37] L. Giovannini, F. Montoncello, and F. Nizzoli, Phys. Rev. B 75, 024416 (2007).

[38] R. Zivieri, P. Malagò, L. Giovannini, S. Tacchi, G. Gubbiotti, and A. O. Adeyeye, J. Phys.: Condens. Matter 25, 336002 (2013).

[39] R. W. Wang and D. L. Mills, Phys. Rev. B 50, 3931 (1994).

[40] R. Zivieri, L. Giovannini, and F. Nizzoli, Phys. Rev. B 62, 14950 (2000).

[41] F. Montoncello, L. Giovannini, F. Nizzoli, P. Vavassori, and M. Grimsditch, Phys. Rev. B 77, 214402 (2008).

[42] Z. Bai, J. Demmel, J. Dongarra, A. Ruhe, and H. van der Vorst (eds.), Templates for the solution of Algebraic Eigenvalue Problems: A Practical Guide (SIAM, Philadelphia, 2000) Chap. 5.

[43] A. Knyazev, in Templates for the Solution of Algebraic Eigenvalue Problems: A Practical Guide, edited by Z. Bai, J. Demmel, J. Dongarra, A. Ruhe, and H. van der Vorst (SIAM, Philadelphia, 2000), Chap. 11.3.
[44] Y. Saad, Iterative Methods for Sparse Linear Systems, 2nd ed. (SIAM, Philadelphia, 2003).

[45] B. A. Ivanov, H. J. Schnitzer, F. G. Mertens, and G. M. Wysin, Phys. Rev. B 58, 8464 (1998).

[46] A. Weinstein, J. Differ. Geom. 18, 523 (1983).

[47] V. V. Dobrovitski, M. I. Katsnelson, and B. N. Harmon, Phys. Rev. Lett. 90, 067201 (2003).

[48] J. F. Cochran and J. R. Dutcher, J. Appl. Phys. 63, 3814 (1988).

[49] J. F. Cochran and J. R. Dutcher, J. Magn. Magn. Mater. 73, 299 (1988).

[50] G. Consolo, G. Gubbiotti, L. Giovannini, and R. Zivieri, Appl. Math. Comput. 217, 8204 (2011).

[51] G. Bertotti, C. Serpico, I. D. Mayergoyz, A. Magni, M. d'Aquino, and R. Bonin, Phys. Rev. Lett. 94, 127206 (2005).

[52] O. Dmytriiev, M. Dvornik, R. V. Mikhaylovskiy, M. Franchin, H. Fangohr, L. Giovannini, F. Montoncello, D. V. Berkov, E. K. Semenova, N. L. Gorn, A. Prabhakar, and V. V. Kruglyak, Phys. Rev. B 86, 104405 (2012).

[53] W. Scholz, Ph.D. thesis, Fakultät für Naturwissenschaften und Informatik, TU Wien, 2003.

[54] M. d'Aquino, C. Serpico, and G. Miano, J. Comp. Phys. 209, 730 (2005)

[55] K. S. Novoselov, A. K. Geim, S. V. Dubonos, E. W. Hill, and I. V. Grigorieva, Nature (London) 426, 812 (2003).

[56] R. Herz and H. Kronmüller, J. Magn. Magn. Mater. 4, 36 (1977).

[57] J. R. Shewchuk, An Introduction to the Conjugate Gradient Method Without the Agonizing Pain, Technical Report, School of Computer Science, Carnegie Mellon University, Pittsburgh, 1994.

[58] W. W. Bradbury and R. Fletcher, Numer. Math. 9, 259 (1966).

[59] M. Frigo and S. G. Johnson, Proc. IEEE 93, 216 (2005).

[60] M. C. Payne, M. P. Teter, D. C. Allan, T. A. Arias, and J. D. Joannopoulos, Rev. Mod. Phys. 64, 1045 (1992).

[61] Y. T. Feng and D. R. J. Owen, Int. J. Numer. Methods Eng. 39, 2209 (1996).

[62] J. H. Mentink, M. V. Tretyakov, A. Fasolino, M. Katsnelson, and T. Rasing, J. Phys.: Condens. Matter 22, 176001 (2010).

[63] A. A. Thiele, Phys. Rev. Lett. 30, 230 (1973).

[64] W. Döring, Z. Naturforsch. 3a, 373 (1948).

[65] N. L. Schryer and L. R. Walker, J. Appl. Phys. 45, 5406 (1974).

[66] J. M. Winter, Phys. Rev. 124, 452 (1961).

[67] M. I. Katsnelson and A. Fasolino, Acc. Chem. Res. 46, 97 (2013).

[68] M. I. Katsnelson, Graphene: Carbon in Two Dimensions (Cambridge University Press, Cambridge, UK, 2012), Chap. 9.

[69] I. M. Lifshitz, Zh. Eksp. Teor. Fiz. 22, 475 (1952).

[70] G. M. Wysin, Phys. Rev. B 54, 15156 (1996).

[71] B. A. Ivanov and V. A. Stephanovich, Phys. Lett. A 141, 89 (1989).

[72] B. A. Ivanov and C. E. Zaspel, Appl. Phys. Lett. 81, 1261 (2002).

[73] S. Mühlbauer, B. Binz, F. Jonietz, C. Pfleiderer, A. Rosch, A. Neubauer, R. Georgii, and P. Böni, Science 323, 915 (2009).

[74] C. Moutafis, S. Komineas, and J. A. C. Bland, Phys. Rev. B 79, 224429 (2009).

[75] N. Burgoyne and R. Cushman, Celest. Mech. 8, 435 (1974).

[76] J. Williamson, Am. J. Math. 58, 141 (1936).

[77] C.-S. Liu, K.-C. Chen, and C.-S. Yeh, J. Mar. Sci. Tech. 17, 228 (2009). 